THE RESEARCH INSTITUTE OF INDUSTRIAL ECONOMICS

Working Paper No. 649, 2005

Oppositional Identities and the Labor Market

by Harminder Battu, McDonald Mwale and Yves Zenou

IUI, The Research Institute of Industrial Economics

P.O. Box 55665

SE-102 15 Stockholm

Sweden 


\title{
Oppositional Identities and the Labor Market*
}

\author{
Harminder Battu ${ }^{\dagger}$ \\ University of Aberdeen \\ McDonald Mwale \\ University of Aberdeen \\ Yves Zenou ${ }^{\S}$ \\ IUI and GAINS, Université du Maine \\ January 10, 2005 (Revised October 19, 2005)
}

\begin{abstract}
We develop a model in which non-white individuals are defined with respect to their social environment (family, friends, neighbors) and their attachments to their culture of origin (religion, language), and in which jobs are mainly found through social networks. We find that, depending on how strong peer pressures are, nonwhites choose to adopt "oppositional" identities since some individuals may identify with the dominant culture and others may reject that culture, even if it implies adverse labor market outcomes.

Key words: Ethnic minorities, identity, social networks, white's norm, multiple equilibria

JEL Classification: A14, J15

${ }^{*}$ We are grateful to the editor, Christian Dustmann, and two anonymous referees for their comments and suggestions. We also thank the seminar participants at the University of Bristol, the 18th Congress of the EEA, Stockholm, the Tinbergen Institute, Amsterdam, and FIEF, Stockholm, for helpful comments, in particular Paul Grout, Gerard van den Berg and Thibaud Vergé. Yves Zenou thanks the Marianne and Marcus Wallenberg Foundation for financial support.

${ }^{\dagger}$ Department of Economics, University of Aberdeen, Edward Wright Building, Dunbar Street, Old Aberdeen AB24 3QY, UK. E-mail: h.battu@abdn.ac.uk

${ }^{\ddagger}$ Health Economics Research Unit (HERU), University of Aberdeen, Medical School, Polwarth Building, Aberdeen AB25 2ZD, UK. E-mail: m.mwale@abdn.ac.uk

${ }^{\S}$ Corresponding author. Address: IUI, The Research Institute of Industrial Economics, Box 55665, 10215 Stockholm, Sweden. E-mail: yvesz@iui.se
\end{abstract}




\section{Introduction}

During the spring and early summer of 2001 there were a series of violent disturbances in various cities and towns in England involving mainly South Asian youth and the police. As a consequence, a number of local and national enquiries were formed to investigate the causes. Though a range of potential explanations were proposed, two received considerable attention both in political circles and in the media. First, the lack of a shared civic identity to bring together diverse communities. Second, increasing segregation of communities on economic, geographic, racial and cultural lines even where this reflected individual preferences.

The interest paid to these two factors is relatively novel in the UK, and does represent a departure from the long-standing debate in the UK which has tended to emphasize racial discrimination as the key force in driving ethnic disadvantage (CRE, 2002). ${ }^{1}$ The debate in the US, at both a policy and academic level, on these types of issues is of longer standing. One theme that has emerged from the academic literature is that some individuals in ethnic groups may "choose" to adopt what are termed "oppositional" identities. Where a community or group is socially excluded from a dominant group, some individuals of that group may identify with the dominant culture and others may reject that culture. ${ }^{2}$ This may occur even if the latter groups preferences involve a lower economic return. From the standpoint of those who choose not to take a rejectionist stance, the rejectionists are making poor economic decisions; they are engaging in self-destructive behavior.

Such preferences may stem from a lack of economic opportunity, discrimination or it may stem from a desire to display greater racial or religious solidarity (Akerlof and Kranton, 2000). Ihlanfeldt and Scafidi (2002) evoke a wish to share culture, prejudice against whites, or expectations of unfavorable treatment by whites against non-whites in white neighborhoods. One could also think of the advantages that members of a minority group can derive from locating close to one another, thereby improving their access to 'ethnic goods' such as food, education or religious service, not to mention the ability to socially interact in their own language. Another related literature focusing on the academic achievement of African American youths argues that African American students in poor areas may be ambivalent about learning standard English, where this may be regarded as "acting white" and adopting mainstream identities (Ogbu, 1978; Fordham and Ogbu, 1986; Delpit, 1995; Ainsworth-Darnell and Downey, 1998, Austen-Smith and Fryer, 2005).

In this paper, we develop a model in which non-white individuals are defined with respect to their social environment (family, friends, neighbors) and their attachments to their culture of origin (religion, language), and in which jobs are mainly found

\footnotetext{
${ }^{1}$ For further details see Building Cohesive Communities (2001) and the Cantle Report (2001).

${ }^{2}$ An alternative explanation revolves around qualifications: skilled minorities could benefit more from integration than unskilled minorities (Cutler and Glaeser, 1997).
} 
through social networks. Non-whites must decide to totally or partially adopt the white's culture or to reject it by anticipating the implications of this choice on their labor market outcomes. Interacting with whites is beneficial because non-white workers may then benefit from the high quality of whites' social networks since the latter do not suffer discrimination. We found that, totally identical individuals can end up with totally different choices. Indeed, depending on the strength of peer pressures, non-whites choose to adopt "oppositional" identities since some of them may identify with the dominant culture and others may reject that culture. We found in particular that some non-whites will totally reject the white's culture even though they know that it will sharply decrease their chance of being employed.

Few theoretical models have investigated the link between ethnic preferences and labor market outcomes. Akerlof (1997) discusses informally a model that has these features whereas Selod and Zenou (2005) essentially focus on the urban consequences (i.e. ghettos) of ethnic preferences. There are also some recent papers that have focussed on the links between identity and education. Akerlof and Kranton (2002) propose a theory in which a student's primary motivation is his or her identity and the quality of a school depends on how students fit in a school's social setting. In an innovative paper, Austen-Smith and Fryer (2005) model peer pressures in education by putting forward the tension faced by individuals between signalling their type to the outside labor market and signalling their type to their peers: signals that induce high wages can be signals that induce peer rejection. One of their main results is to show that the more individuals discount the future, the more acute peer pressure becomes and the more homogeneous groups are (in terms of education).

\section{The theoretical model}

In this section, we would like to derive a simple model showing that ethnic preferences (the desire or reluctance to interact with individuals of other ethnic groups) can have strong implications in the labor market. There are two stages. In the first one, non-white individuals decide to adopt the white's norm or not anticipating the implications of this choice on their labor market outcomes. This is the second stage. Before describing each stage, we will first set out the utilities of the workers and how workers obtain a job.

\subsection{Ethnic preferences and utilities}

There is a finite number of non-white and white individuals, which are respectively given by $N_{N W}$ and $N_{W}$, with $N_{N W}+N_{W}=N$. We assume that $N_{W}>N_{N W}$, which is the case in most areas (cities, regions, etc.) in developed countries. Whites and nonwhites are totally identical; they just differ by an observable trait, which is the color of their skin. The social space is the interval $[0,1]$. For simplicity, the white's 
norm is normalized to zero and all other workers (non-whites) define themselves with respect to this norm.

As we will see below, non-white workers optimally choose their "location" $0 \leq$ $x \leq 1$ in the social space. In this interval, there are two extreme locations: $x=0$ means to totally adopt the white's norm and thus to totally reject the norm of the ethnic group the worker belongs to, whereas $x=1$ implies the contrary (to totally reject the white's norm and thus to totally adopt the norm of the ethnic group the worker belongs to). Any location's choice of $0<x<1$ leads to a behavior which is in between these two extremes. Thus, the larger $x$ the more distant the worker is from the white's norm and the closer he/she is to his/her own community.

Available data does indeed reveal a range of preferences and hence locations across social space for different ethnic group members. For example, the UK Fourth National Survey of Ethnic Minorities (FNSEM) undertaken in 1993/94 asks ethnic respondents about their identification with Britishness, attitudes towards intermarriage and preferences in terms of the ethnic makeup of their own child's school. This information can be used to locate individual ethnic groups in social space $(x)$ relative to the white majority. Table 1 reveals that nearly three-quarters of African-Asians thought of themselves as British compared to only just over a half of Bangladeshis. ${ }^{3}$ The Caribbeans on this measure seem to be the furthest from the white majority with one-third likely to disagree with being British. This contrasts with the West Indian migrants of the 1940s and 1950s who by most accounts thought of themselves as British and often talked of coming to "the mother country" (Modood et al. 1997). The Chinese in Table 1 stand out since roughly equal percentages agreed and disagreed with the notion of being British ( $44 \%$ and $41 \%$ respectively). On this dimension at least the Chinese seem to sit at both extremes in terms of their location in social space.

Table 2 provides some data on another dimension of identity, namely marriage and in particular attitudes to inter-marriage. Inter-marriage can be considered a measure of social assimilation and also a factor producing it (Pagnini and Morgan, 1990). On the other hand some ethnic and religious groups regard inter-ethnic marriage as a potential threat endangering and undermining ethnic identities. In the FNSEM individuals were asked "If a close relative were to marry a white person would you not mind, would you mind a little, would you mind very much?" Here significant percentages of the three South-Asian groups said they would mind very much with the greatest hostility being among the Pakistani population (37\% of them say they would mind very much a mixed marriage). On this dimension significant numbers of South-Asians display low levels of social assimilation and are close to 1 in social space. A majority of the other groups said that they would not mind and

\footnotetext{
${ }^{3}$ The African-Asians arrived in the UK from the late 1960s following persecution and forced expulsion from a number of East African countries.
} 
amongst Caribbeans, African-Asians and the Chinese the percentages that would mind very much are quite small. ${ }^{4}$ The evidence for the Caribbean population is somewhat unsurprising given that in 1991 27\% of married or cohabiting Caribbean males had a white partner (Peach, 2005).

\section{[Insert Tables 1 and 2 here]}

Tables 3 and 4 relate to an important area of controversy both in the UK and US; the role of schools in keeping different ethnic communities apart. Individuals in the FNSEM were asked about the importance of ethnicity in choosing a school (Table 3) and the proportion of one's ethnic group they would like in their own children's school (Table 4). There are indirect ways to distance oneself from the white culture since separate schooling makes the interaction with whites more difficult. The ethnic makeup of a school was deemed an important consideration for around one-third of Caribbean's, Bangladeshis and Pakistanis. Less than a quarter of African-Asians and Indians and only one in ten Chinese thought this important. Table 4 gives some data on the preferred proportion of one's ethnic group in a school. Of those who did have a preference over a third of Caribbeans, Pakistanis and Bangladeshis wanted a school with half or more from their own ethnic group. For African-Asians and Indians the equivalent figures are lower and for the Chinese considerably lower.

\section{[Insert Tables 3 and 4 here]}

On these dimensions then African-Asians and the Chinese have an $x$ closer to 0 . They are socially assimilated and economically successful (Peach, 1996). The Indian population seems to stand somewhere in the middle having done well economically but are socially more closed than African-Asians and the Chinese. The Pakistani and Bangladeshi populations exhibit a more economically marginal position and do display greater social distance from the majority community. The Caribbean population represents somewhat of a quandary. On many dimensions they are socially assimilated (language, inter-marriage, spatial segregation) but they are economically poor (Peach, 2005). Their assimilation seems to generate little economic payoff.

Let us now describe formally the preferences of whites and non-whites, who are all assumed to be risk neutral. Since whites are located at $x=0$, the instantaneous (indirect) utility function of a white worker of employment status $j=U, E$ is given by:

$$
V_{W j}=y_{j}
$$

\footnotetext{
${ }^{4}$ Hostility to inter-marriage may not always be a signal of an oppositional identity. For example, if one believes in assimilation but also believes that society is prejudiced against inter-racial partnerships (or the children of such partnerships) one may be hostile to a close relative marrying outwith ones own community but still in favour of mainstream culture.
} 
where $y_{j}$ is the exogenous income of a worker with employment status $j\left(y_{E}\right.$ and $y_{U}$ are respectively the wage of the employed and the unemployment benefit, with $\left.y_{E}>y_{U}>0\right)$.

All nonwhites are totally identical ex ante. Thus, the instantaneous (indirect) utility function for a non-white worker $i=1, \ldots, N_{N W}$ of employment status $j=$ $U, E$, and "location" $0 \leq x_{i} \leq 1$, is given by: ${ }^{5}$

$$
V_{N W j}\left(x_{i}\right)=y_{j}+e x_{i} \bar{x}_{N W}
$$

where $\bar{x}_{N W}=\frac{1}{N_{W}-1} \sum_{j=1}^{j=N_{W}-1} x_{j}$ is the average or aggregate identity choice of all nonwhite workers but $i$, and $e>0$ the intensity of peer effects. In this formulation, non-whites define themselves with respect to whites $\left(x_{i}\right)$ and to their peers $\left(\bar{x}_{N W}\right){ }^{6}$

This utility function has two parts. The first part expresses the fact that this utility increases in income $y_{j}$. The second part concerns the product between own action $x_{i}$ and group action $\bar{x}_{N W}$. The utility reaches its maximum (equal to $y_{j}+e$ ) if both the individual non-white and all other non-whites are not assimilated at all (i.e. $x_{i}=\bar{x}_{N W}=1$ ). It reaches its minimum (equal to $y_{j}$ ) if either the individual non-white or the non-white group is completely assimilated $\left(x_{i}=0\right.$ or $\left.\bar{x}_{N W}=0\right)$ or both are completely assimilated $\left(x_{i}=\bar{x}_{N W}=0\right)$. The left panel of Figure 1 illustrates this utility function for different values of $x_{i}$. The right panel of Figure 1 shows different indifference curves where utility increases when moving upward.

\section{[Insert Figure 1 here]}

One of the crucial assumptions is that utility increases in the degree of nonassimilation of one's ethnic group $\left(\partial V_{N W j} / \partial \bar{x}_{N W}=e x_{i} \geq 0\right)$. This presupposes that non-whites have some a priori reluctance to be like whites and they would always prefer to be as far as possible from the white's norm and culture. This is admittedly a strong assumption but it is adopted because we want to show that, even with extreme negative preferences like this one, there is still some possibility (as we will see below) that, in equilibrium, some non-whites will choose to totally assimilate to the whites, i.e. $x_{i}=0$. In fact we will show (see Proposition 1 below) that, with preferences described by (2), there will always be an equilibrium with $x_{i}=\bar{x}_{N W}=0$, for all $i=1, \ldots, N_{N W}$. Let us now justify why these preferences, though extreme, are not so unrealistic.

\footnotetext{
${ }^{5}$ This is a standard way of modelling strategic complementarities (see e.g. Diamond, 1981 or Vives, 1999) since$$
\frac{\partial^{2} V_{N W j}}{\partial x_{i} \partial \bar{x}_{N W}}=e>0
$$

which means that the higher the group identity effort, the higher the identity effort of $x_{i}$.

${ }^{6}$ Even though the focus and the model are totally different, Fershtman and Weiss (1998) have a similar group externality that affects the utility of each individual.
} 
First, a natural argument revolves around the transmission of preferences as for example in Bisin et al. (2004). For example, in the United States, because AfricanAmerican history is one of slavery and discrimination, black parents may be inclined to transmit this lack of trust for the whites to their offspring (see, for example, Alesina and La Ferrara, 2002, for an interesting study on trust and racial mixing). ${ }^{7}$ This may also be evident among immigrants in Europe, especially among the Muslim community and reflect discrimination and hostility upon arrival. Individuals are then a priori better off by affirming their identity and to reject the white's norm. The flipside of this is the "acting white" phenomenon, well-known by sociologists and psychologists (Fordham and Ogbu, 1986, Ainsworth-Darnell and Downey, 1998) and recently studied by economists (Austen-Smith and Fryer, 2005). If you or your group tend to be assimilated, then your utility decreases because of peer pressure.

Second, because there are cultural and sometimes language and religious differences between whites and non-whites, this utility expresses the fact that there is a cost for non-whites in interacting with whites. In (2), for a given $\bar{x}_{N W}$, this cost is captured indirectly through the distance $x_{N W}$ in social space. It is easy to see that when the distance to the white community increases, utility increases, reflecting the disutility of interracial contacts with white "neighbors".

Third, because peer pressures do matter, the utility of nonwhites positively depends on $\bar{x}_{N W}$ the (aggregate) choice of the other nonwhites. Indeed, for a given $x_{N W}$, the more your peers choose to distant themselves from whites, the higher is your utility. ${ }^{8}$ Take two extreme cases. If all your peers choose to totally reject whites' values, i.e. $\bar{x}_{N W}=1$, then your instantaneous utility is $y_{j}+e x_{i}$, so that only your location choice is affecting you. If, on the contrary, all your peers choose to totally adopt the white's norm, i.e. $\bar{x}_{N W}=0$, then your choice does not matter since your utility is just your income $y_{j}$. As a result, $0 \leq \bar{x}_{N W} \leq 1$ and thus the choice of your peers always reduces the impact of your own choice on your utility. Of course, the magnitude of this reduction depends on $e$, which can thus be interpreted as the importance of peer effects and social environment: if $e$ is very high (it could be greater than one), then peers have a strong effect on the choice of $x_{i}$. In other words, depending on its value, $e$ can amplify or reduce the effects of the peers. There is thus a group externality that is captured by $\bar{x}_{N W}$ since when a worker choose $x_{i}$, he/she influences the choice of his/her peers.

To summarize, the utility function (2) captures the tension between own identity and group identity choices, with a negative biased towards assimilation. A stated

\footnotetext{
${ }^{7}$ The movie by Spike Lee "Do the Right Thing" is a good example of this lack of trust between black and white communities that is transmitted from one generation to another.

${ }^{8}$ In his study about religious groups, Berman (2000) has a similar externality. In his model, the more your peers do the same activity as you, the higher is your utility (for example, praying is much more satisfying the more participants there are). Here, what matters is the average choice of your peers rather that the number of your peers who make the same choice.
} 
above, the maximum level the utility can reach is when both the individual and the reference group choose not to assimilate, i.e. $x_{i}=\bar{x}_{N W}=1$. The lowest level it can reach is when both choose to totally assimilate, i.e. $x_{i}=\bar{x}_{N W}=0$. This is where the biased is since, even if the individual and the group make the same choice, it can lead to the lowest possible utility level because it is "wrong" to totally assimilate to the whites (the ethnic culture will be totally lost). We strongly believe that, for ethnic minorities, there is cultural transmission from the parents and the family to the children that emphasizes the importance of preserving one's own ethnicity and to reject the dominant culture. For example, it is commonly observed that ethnic parents disapprove interracial marriage (in Table 2, for Indians, Pakistanis and Bangladeshis, between 27 to 36 percent will mind very much is a close relative were to marry a white person).

Now imagine that we assume a standard "conformist" utility function where there is cost from failing to conform to others (see, among others, Akerlof, 1980, Akerlof, 1997, Ballester et al., 2005, Bernheim, 1994, Kandel and Lazear, 1992, Fershtman and Weiss, 1998, Patacchini and Zenou, 2005). It is given by:

$$
V_{N W j}\left(x_{i}\right)=y_{j}+c x_{i}^{2}-d\left(x_{i}-\bar{x}_{N W}\right)^{2}
$$

where we assume that $d>c>0$. This condition guarantees that $V_{N W j}\left(x_{i}\right)$ is strictly concave in $x_{i}$, i.e. $V_{N W j}^{\prime \prime}\left(x_{i}\right)<0$. Apart from the revenue $y_{j}$, this utility has two parts. The first one, $c x_{i}^{2}$, is the utility obtained by individual $i$ from choosing identity $x_{i}$ when $i$ 's friends do not have any impact on $i$ 's decision. The second part captures the influence of friends' behavior on own action. It is such that each individual wants to minimize the social distance between him/herself and his/her reference group, where $d$ is the parameter describing the taste for conformity of individual $i$. Observe that, in this case, utility does not necessarily increase in the degree of non-assimilation of one's ethnic group since $\partial V_{N W j} / \partial \bar{x}_{N W}=-2 d\left(x_{i}-\bar{x}_{N W}\right)$, which can be positive or negative depending on the distance in terms of identity between $i$ and his/her friends. At the end of section 3, we calculate the different equilibria with (3) and show that the results are identical to the case where the utility function is given by (2).

In order to investigate further this issue of the choice of the utility function, in Appendix B, we consider the case when someone's utility increases the more assimilated his/her ethnic group. We choose the following preferences:

$$
V_{N W j}\left(x_{i}\right)=y_{j}+e x_{i}\left(K-\bar{x}_{N W}\right)
$$

where $K>1$. As in (2), the utility reaches its minimum when $x_{i}=0$ (and/or $\bar{x}_{N W}=0$ ) but reaches its maximum when $x_{i}=1$ and $\bar{x}_{N W}=0$, i.e. the individual totally rejects the white's norm while the group is completely assimilated. This presupposes that there is a negative bias towards assimilation at the individual level 
(i.e. $\left.\partial V_{N W j} / \partial x_{i}=e\left(K-\bar{x}_{N W}\right)>0\right)$ but a positive bias at the group level (i.e. $\left.\partial V_{N W j} / \partial \bar{x}_{N W}=-e x_{i}<0\right)$. Observe also that $\frac{\partial^{2} V_{N W j}}{\partial x_{i} \partial \bar{x}_{N W}}=-e<0$, which means that the higher the group identity effort, the lower the identity effort of $x_{i}$ ( $x_{i}$ and $\bar{x}_{N W}$ are strategic substitute).

\subsection{Social networks and the job acquisition rate}

Let us now describe the way the labor market operates. Here we focus on jobs that are available to both whites and non-whites, i.e. jobs for which whites and nonwhites compete for. This means that we are not interested in self-employment and in jobs that are only available to non-whites (because for example it implies knowing the language of the community).

At any moment of time, workers can either be employed or unemployed. We assume that changes in employment status (employment versus unemployment) are governed by a continuous-time Markov process. Firms are assumed to use "local" or informal methods so that jobs can mainly be obtained through word-of-mouth communications (for example firms do not advertise their vacancies but transmit the information about them only to their employed workers, who, in turn, give this information to their "friends"). In our framework, there is a two-stage procedure to obtain a job. First, workers must have a job contact with a firm (through their social network) and then a job match with this firm (as for example in Pissarides, 2000, ch.6). The first stage requires that unemployed workers acquire information about jobs (this process will be detailed below) in order to establish a contact. In the second stage, the match is automatically realized for whites, whereas it is realized with probability $m<1$ for any non-white worker. This is because we assume that there are two types of firms in the economy: non-discriminating firms (in proportion $m$ ) and discriminating firms (in proportion $1-m$ ). So when a non-white worker has a contact with a firm, this job contact is transformed into a job match only if the firm does not discriminate against non-whites. The probability $1-m$ can represent the prejudices of employers who dislike associating with non-white workers (Becker, 1957). Observe that $m$ does not depend on $x_{i}$. This means that labor market discrimination is not affected by the norm that a non-white adopts. In other words, if a non-white chooses to totally adopt the white's culture $\left(x_{i}=0\right)$, he/she will be seen by a discriminatory employer exactly as any other non-white that has chosen to totally reject the white's culture $\left(x_{i}=1\right) .^{9}$

We assume that job contacts randomly occur at an endogenous rate $\theta_{W}$ for whites and $\theta_{i}\left(x_{i}\right)$ for a non-white worker located at a "distance" $x_{i}$ from the white's norm

\footnotetext{
${ }^{9}$ It is easy to generalize the model by having $m\left(x_{i}\right)$, with $0<m\left(x_{i}\right)<1$ and $m^{\prime}\left(x_{i}\right)<0$, so that employers discriminate more against non-whites that have chosen to distance themselves from the white's norm. However, this will complicate the analysis without changing the qualitative results of our main Proposition (Proposition 2 below) because the effects will be even stronger.
} 
while the exogenous job separation rate is $\delta$. In this context, the job acquisition rate (that is the transition rate from unemployment to employment) is the product of the job contact rate and the probability of a job match. Since whites always transform a job contact into a job match, their job acquisition rate is equal to their job contact rate $\theta_{W}$. For non-whites, the job contact rate must be multiplied by $m$ (the probability that the contacted firm is not discriminating).

Let us now determine the job contact rate for all workers. For a white worker, we have

$$
\theta_{W}=\mu+\lambda s_{W}
$$

whereas, for a non-white worker located at a "distance" $x_{i}$ from the white's norm, it is given by:

$$
\theta_{N W}\left(x_{i}\right)=\mu+\lambda s_{N W}\left(x_{i}\right)
$$

where $\mu>0$ is the common information about jobs available to anyone (independently of race or space), $s_{W}$ and $s_{N W}\left(x_{i}\right)$ denote the local social network of respectively whites and non-white workers located at $x_{i}$, and $\lambda$ is a positive parameter that measures the impact of social network on the job contact rate.

In the specification we have chosen, the job contact rate only depends on the amount of information workers can gather about job opportunities. Formulas (5) and (6) assume that a given level of information is available to anyone and that this level of information may be altered through social networks. In other words, besides the common knowledge factor, there is another way of learning about jobs: employed workers hear about the job on the workplace and transmit this information to their "friends".

Let us now define what we mean by friends and social networks. The local connections that whites and non-whites can use to find a job are respectively measured by $s_{W}$ and $s_{N W}\left(x_{i}\right)$, which we assume to be a positive function of that group's employment rate, i.e. respectively $1-u_{W}$ and $1-u_{N W}$. In other words, when the unemployment rate is high among a particular group, individuals of that group have few connections that can refer them to jobs and their social network is poor (Calvó-Armengol, 2004, Calvó-Armengol and Jackson, 2004, Calvó-Armengol and Zenou, 2005, Montgomery, 1991, Mortensen and Vishwanath, 1994, Topa, 2001). This is because, in our model, only the employed can transmit information about jobs. In this respect, the employment rate measures the quality of a group's social network.

For a worker of type $k=N W, W$, the social network is given by (remember that the total population is normalized to 1$)$ :

$$
s_{k}\left(x_{i}\right)=\alpha\left(x_{i}\right)\left(1-u_{W}\right) N_{W}+\left(1-\alpha\left(x_{i}\right)\right)\left(1-u_{N W}\right) N_{N W}
$$

with $\alpha\left(x_{i}\right) \in[0,1], \forall x_{i}$, and $\alpha^{\prime}\left(x_{i}\right)<0$. Thus, depending on his/her position $x_{i}$ in 
the social space, each individual benefits more or less of the social networks of all the other workers.

For whites, since $x=0$, we have:

$$
s_{W}=\alpha(0)\left(1-u_{W}\right) N_{W}+(1-\alpha(0))\left(1-u_{N W}\right) N_{N W}
$$

For simplicity and without loss of generality, we assume that

$$
\alpha\left(x_{i}\right)=1-x_{i}
$$

which implies that $\alpha(0)=1$ and $\alpha(1)=0$. As a result, the white's social network is given by:

$$
s_{W}=\left(1-u_{W}\right) N_{W}
$$

which means that their social network only depends on their own employment rate.

For non-whites, the social network will partly depend on their location in the social space. Indeed, non-whites benefit from their own connections to jobs (i.e. their own employment rate) and also from part of the social network of whites. We have: ${ }^{10}$

$$
s_{N W}\left(x_{i}\right)=\left(1-x_{i}\right)\left(1-u_{W}\right) N_{W}+x_{i}\left(1-u_{N W}\right) N_{N W}
$$

The following comments on (9) are in order. First, $s_{N W}\left(x_{i}\right)$ explicitly takes into account the underlying population shares of whites and nonwhites and thus gives a weighted average measure of social distance. Second, two different social networks affect the social network of non-white workers: the white's social network, $1-u_{W}$, and the non-white's one, $1-u_{N W}$. The relative weight of each of them strongly depends on the choice of $x_{i}$ in the social space.

The general idea here is that, the more time one spends with the white community, the less time he/she spends with his/her own community. In a spatial context, this will be even more true since non-whites living in predominately nonwhite (white) neighborhoods will (not) interact very much with other non-whites because of the physical separation between communities. What is crucial here is that there is an externality of being "close" to whites. This externality causes the employment rate of non-whites to be positively affected by the employment rate of whites. However, depending on the value of $x_{i}$ (the willingness to interact with whites or to adopt the white's culture), non-whites can benefit more or less from whites' connections to jobs.

If, as we will see below, whites have the best connections to jobs (because there are less discriminated against since most of the employers, both in the UK and the US and elsewhere, are whites), then equations (8) and (9) capture the fact that

\footnotetext{
${ }^{10}$ Observe that the social network of an individual of type $i$ here depends both explicitly on his/her own identity choice $x_{i}$ and implicitly on the indentity choice of other co-ethnics through $u_{N W}$, which is, as we will see below, a function of $x$ (see equation (15) below) .
} 
there is a cost (in terms of labor market outcomes) to live in a predominantly white society and not willing to adopt the white's norm (i.e. being oppositional).

\section{The (two-stage) equilibrium}

As stated above, there are two stages. In the first stage, non-white workers choose their location $x$ in the social space (we have imposed the location $x=0$ for whites). In the second stage, the labor market outcomes (i.e. the unemployment rate and the probability to find a job) of each white and each non-white are determined. Because of backward induction, we solve the second stage first.

We have seen that changes in the employment status of white and non-white workers are governed by a time continuous Markov process in which $\theta_{W}$ and $m \theta_{N W}(x)^{11}$ are respectively the group-specific transition rate (defined by (5) and (6)) and $\delta$ is the job destruction rate. As a result, plugging (8) in (5), the probability to find a job for whites is equal to

$$
\theta_{W}=\mu+\lambda\left(1-u_{W}\right) N_{W}
$$

whereas, for non-whites, by plugging (9) in (6) and using (7), it is given by:

$$
m \theta_{N W}(x)=\mu m+\lambda m\left[(1-x)\left(1-u_{W}\right) N_{W}+x\left(1-u_{N W}\right) N_{N W}\right]
$$

Since each job is destroyed according to a Markov process with arrival rate $\delta$, then the number of workers of type $k=N W, W$ who enter unemployment is $\delta\left(1-u_{k}\right) N_{k}$ and the number who leave unemployment is $m_{k} \theta_{k} u_{k} N_{k}$, with $m_{W}=1$ and $m_{N W}=m<1$. The evolution of unemployment is thus given by the difference between these two flows,

$$
\left(u_{k} N_{k}\right)=\delta\left(1-u_{k}\right) N_{k}-m_{k} \theta_{k} u_{k} N_{k} \quad, \quad k=N W, W
$$

where $\left(u_{k} N_{k}\right)$ is the variation of unemployment with respect to time for workers of type $k$. In steady state, the level of unemployment is constant and therefore these two flows are equal (flows out of unemployment equal flows into unemployment). We thus have:

$$
u_{k}=\frac{\delta}{\delta+m_{k} \theta_{k}} \quad, \quad k=N W, W
$$

Thus, for whites, we have:

$$
u_{W}=\frac{\delta}{\delta+\theta_{W}}
$$

\footnotetext{
${ }^{11}$ When there is no possible confusion, we will omit the index $i$ for $x$.
} 
whereas, for non-whites with location $x$, we obtain:

$$
u_{N W}(x)=\frac{\delta}{\delta+m \theta_{N W}(x)}
$$

where $u_{N W}(x)$ denotes the unemployment rate of non-white workers as a function of $x$ the location in the social space.

Lemma 1 in the Appendix shows that we have $u_{W}<u_{N W}$ whatever the location chosen in the first stage by non-whites. Lemma 2 in the Appendix shows that when $\mu>\lambda$, the solutions of (14) and (15) are unique, strictly positive, strictly between 0 and 1 , and respectively given by (18), (19) and (20).

Let us now solve the first stage of the model. We can now calculate the expected utilities of each group. To do that, we assume perfect capital markets with a zero interest rate, ${ }^{12}$ which enable workers to smooth their income over time as they enter and leave unemployment: workers save while employed and draw down on their savings when out of work. At any moment, the disposable income of a worker is thus equal to that worker's average income over the job cycle. Therefore, using (1), the expected utility of a white worker is equal to

$$
\begin{aligned}
E V_{W} & =\left(1-u_{W}\right) V_{W E}+u_{W} V_{W U} \\
& =y_{E}-u_{W}\left(y_{E}-y_{U}\right)
\end{aligned}
$$

For a non-white worker $i$ located in $x_{i}$, using (2), it is given by:

$$
\begin{aligned}
E V_{N W}\left(x_{i}\right) & =\left(1-u_{N W}\left(x_{i}\right)\right) V_{N W E}\left(x_{i}\right)+u_{N W}\left(x_{i}\right) V_{N W U}\left(x_{i}\right) \\
& =y_{E}+e x_{i} \bar{x}_{N W}-u_{N W}\left(x_{i}\right)\left(y_{E}-y_{U}\right)
\end{aligned}
$$

where $u_{N W}\left(x_{i}\right)$ is determined by (15).

We restrict our attention to symmetric equilibria such that all agents choose the same $x$. We have the following definition:

Definition 1 A steady-state market equilibrium is a vector $\left(x_{N W}^{*}, \bar{x}_{N W}^{*}, u_{W}^{*}, u_{N W}^{*}\right)$ such that:

(i) The choice $x_{N W}^{*}$ of each nonwhite maximizes his/her expected utility $E V_{N W}(x)$ anticipating the resulting labor market outcomes and taking as given the aggregate choice of all other nonwhites $\bar{x}_{N W}^{*}$.

\footnotetext{
${ }^{12}$ When there is a zero interest rate, workers have no intrinsic preference for the present so that they only care about the fraction of time they spend employed and unemployed. Therefore, the expected utilities are not state dependent. For example, since a white worker spends a fraction $\theta_{W} /\left(\theta_{W}+\delta\right)$ of his lifetime employed and a fraction $\delta /\left(\theta_{W}+\delta\right)$ unemployed, his average income is equal to $\frac{\theta_{W}}{\theta_{W}+\delta} y_{E}+\frac{\delta}{\theta_{W}+\delta} y_{U}$. The same analysis applies for non-whites.
} 
(ii) The aggregate choice of all nonwhites $\bar{x}_{N W}^{*}$ is consistent with the optimal choices of all nonwhites. Specifically, $\bar{x}_{N W}^{*}=x_{N W}^{*}$.

(iii) The unemployment rates of whites and nonwhites are determined by the steadystate conditions (14) and (15).

We can now fully characterize the steady-state labor market equilibrium. We have our first straightforward result:

Proposition 1 Assume $\mu>\lambda$. Then whatever the values of all other parameters, there always exists a steady-state equilibrium in which all nonwhite workers choose to totally adopt the white's norm, i.e. $x_{N W}^{*}=\bar{x}_{N W}^{*}=0$. Their unemployment rate $u_{S S} \equiv u(0)$ is given by (19).

Proof. The first order condition of (17) is given by:

$$
\frac{\partial E V_{N W}\left(x_{i}\right)}{\partial x_{i}}=e \bar{x}_{j}-u_{N W}^{\prime}\left(x_{i}^{*}\right)\left(y_{E}-y_{U}\right)
$$

where $u_{N W}^{\prime}\left(x_{i}^{*}\right)>0$ (see Lemma 3 in the Appendix). Assume that $\bar{x}_{-i}=0$, then the optimal choice for $i$ is $x_{i}^{*}=0$ since $\frac{\partial E V_{N W}\left(x_{i}\right)}{\partial x_{i}}<0$. Thus, all workers coordinate themselves on this equilibrium and no one has the incentive to deviate.

This result is very intuitive. When workers choose $x_{N W}$ they trade off the gains (higher chance to get a job) with the costs of being close to $x$ (depending on the choice of the others). Now, if all your peers decide to totally adopt the white's norm, it is clear that you will also make the same choice since there are only gains from it (higher chance to get a job and positive externality from the group). Let us now give our general result.

Proposition 2 Assume $\mu>\lambda$. By using the value of $u^{\prime}(0)$ and $u^{\prime}(1)$ in (25) and (26), we have:

(i) If e/( $\left.y_{E}-y_{U}\right)<u^{\prime}(0)$, there is a unique stable steady-state equilibrium in which all nonwhite workers choose to totally adopt the white's norm, i.e. $x_{N W}^{*}=0$ (Figure 1).

(ii) If $u^{\prime}(0)<e /\left(y_{E}-y_{U}\right)<u^{\prime}(1)$, generically two cases may arise:

(iia) if $u^{\prime}(0)$ is large enough, there is a unique stable steady-state equilibrium in which all nonwhite workers choose to totally adopt the white's norm, i.e. $x_{N W}^{*}=0$ (Figure 2). 
(iib) if $u^{\prime}(0)$ is small enough, there are three stable steady-state equilibria (Figure 3). In the first one, all nonwhite workers choose to totally adopt the white's norm, i.e. $x_{N W}^{*}=0$. In the second one, all nonwhite workers choose to partially adopt the white's norm, i.e. $0<\underline{x}_{N W}^{*}<1$ but the value of $\underline{x}_{N W}^{*}$ is quite low. Their unemployment rate $\underline{u}_{I N} \equiv u_{N W}\left(\underline{x}_{N W}^{*}\right)$ is given by (21). In the third one, all nonwhite workers choose to partially adopt the white's norm, i.e. $0<\bar{x}_{N W}^{*}<1$ but the value of $\bar{x}_{N W}^{*}$ is quite high: $\underline{x}_{N W}^{*}<\bar{x}_{N W}^{*}<1$. Their unemployment rate $\bar{u}_{I N} \equiv u_{N W}\left(\bar{x}_{N W}^{*}\right)$ is given by (21).

(iii) If e/( $\left.y_{E}-y_{U}\right)>u^{\prime}(1)$, there are three stable steady-state equilibria (Figure 4). In the first one, all nonwhite workers choose to totally adopt the white's norm, i.e. $x_{N W}^{*}=0$. In the second one, all nonwhite workers choose to totally reject the white's norm, i.e. $x_{N W}^{*}=1$. Their unemployment rate $u_{C O} \equiv u(1)$ is given by (20). In the third equilibrium, all nonwhite workers choose to partially adopt the white's norm, i.e. $0<\widehat{x}_{N W}^{*}<1$, with $\underline{x}_{N W}^{*}<\widehat{x}_{N W}^{*}<\bar{x}_{N W}^{*}$. Their unemployment rate $\widehat{u}_{I N} \equiv u_{N W}\left(\widehat{x}_{N W}^{*}\right)$ is given by (21).

The unemployment rate of whites $u_{W}$ is given by (18). We also have that:

$$
u_{W}<u_{S S}<\underline{u}_{I N}<\widehat{u}_{I N}<\bar{u}_{I N}<u_{C O}
$$

Proof. See Appendix A.

Figures 2a-2d illustrate the different cases. This proposition shows that ex ante identical workers can end up choosing oppositional identities. For example, in case (iii), for exactly the same parameter values, all workers can choose either to totally reject the white's norm $\left(x_{N W}^{*}=1\right)$ or to totally assimilate to it $\left(x_{N W}^{*}=0\right)$. There is a coordination problem in which the group pressure creates externalities in such a way that workers choose oppositional identities. Of course, our results depend on the value of $e$ (the intensity of peer pressure), the wage premium of being employed, $y_{E}-y_{U}$, and the marginal impact of $x$ on the nonwhite unemployment rate $u_{N W}^{\prime}(x)$. To be more precise, there are two forces that counteract each other. On the one hand, non-whites would like to reject the white's norm because it is costly to interact with whites, but, on the other, they are attracted to whites because of the positive consequences in the labor market. Now depending on the choices of the peers, one force can dominate the other.

Proposition 2, case $(i)$ (Figure 2a), shows that if there are low peer pressures (low $e$ ), the payoffs to interact with whites are very high and discrimination $m$ is not too strong (high $\left.\left(y_{E}-y_{U}\right) u^{\prime}(0)\right)$, then all workers will choose to assimilate to the white culture $x_{N W}^{*}=0$. It is clear in this case that no worker will deviate from this equilibrium because the gains are very high and there is basically no cost since the group provides very positive externalities. In terms of the ethnic groups discussed 
earlier, the African-Asians and the Chinese are more likely to be in this type of equilibrium (they are economically successful and socially well assimilated) (Peach, 1996).

At the other extreme, Proposition 2, case (iii) (Figure 2d), shows that if there are strong peer pressures, low rewards to interact with whites and high discrimination, then there are two other equilibria in which all workers will choose to either totally or partially reject the white's norm. This means that, even if it implies a penalty in terms of finding a job, because of strong peer pressures nonwhites reject the white's norm by choosing a $x_{N W}^{*}$ different to zero. The Pakistani and Bangladeshi population since they display greater social distance from the majority community and are economically disadvantaged would seem to be consistent with those who choose $x_{N W}^{*}=1$ (Heath, 2001).

Finally, in the intermediate case where peer pressures and payoffs to interact with whites have intermediate values (Proposition 2, cases (iia) and (iib), Figures $2 \mathrm{~b}$ and $2 \mathrm{c}$ ), other equilibria can emerge in which nonwhites either partially or totally adopt the white's norm. In this case, they will never totally reject the white's norm because the rewards are not too low. The Indian population which has done well economically but is socially more closed than the African-Asians and the Chinese would seem to fit with this intermediate case. The Caribbean population is more difficult to place. In terms of language fluency, inter-marriage and geographical location they are more socially assimilated but still they are economically poor (Peach, 2005).

[Insert Figures $2 a, 2 b, 2 c$ and $2 d]$

An interesting result is that, even if all individuals in a community would like to reject the white's norm (i.e. $x_{N W}^{*}=1$ ), it is not always an equilibrium. In fact, it has to be that peer pressures and discrimination are sufficiently strong and the rewards from interacting with whites sufficiently low (case (iib)). Otherwise, individuals cannot coordinate themselves on this equilibrium in which everybody chooses $x_{N W}^{*}=1$.

Throughout language and also religion play a role in defining and differentiating individuals. As a result, Proposition 2 indicates that non-whites who have different language and religion than that of the majority group (whites) and are strongly attached to them (strong peer pressures) can totally reject the white's norm and are thus more likely to experience adverse labor outcomes. There is an important literature that shows that the lack of fluency in the English language has indeed adverse effects on both assimilation and labor market outcomes of non-white workers (especially immigrants). This literature begins with Chiswick (1978) and has been studied further by, among others, McManus et al. (1983) and Borjas (1994) for the US, and Dustmann and Fabbri (2003) for the UK. Indeed, the Dustmann and Fabbri (2003) study finds that Pakistanis and Bangladeshis in the UK do have 
a lower level of language fluency compared to other ethnic groups. Concerning religion, there is a small literature on the economic consequences of religion (see in particular Iannaccone, 1998) but, to our knowledge, not on the impact of religion on the degree of assimilation and labor market outcomes of immigrants. A notable exception is Lazear (1999) who focuses on cultural differences (religion is obviously part of the culture of people) between the minority and the majority group. He shows that individuals from minority groups are more likely to adopt the culture of the majority when the minority group accounts for a small proportion of the total population.

It is now interesting to investigate the case of "conformist" preferences given by (3) with $d>c>0$. In fact, by focusing on symmetric equilibria $\left(x_{i}=\bar{x}_{N W}\right)$ and observing that Lemmata 1, 2, 3, 4 and 5 are still valid, and by replacing $e$ by $2 c$ in the proof of Proposition 2, so that $g(x) \equiv \frac{2 c}{y_{E}-y_{U}} x$, we exactly obtain Proposition 2 . There is still a negative bias towards assimilation since, in a symmetric equilibrium, $\partial V_{N W j} / \partial x_{i}=2 c x_{i}>0$, so that the less someone is assimilated, the better off he/she is. Observe that multiple equilibria emerge mainly because $x_{i}$ and $\bar{x}_{N W}$ are strategic complement $\left(\partial^{2} V_{N W j} / \partial x_{i} \partial \bar{x}_{N W}>0\right)$.

In the opposite case when the utility function is given by (4), that is when someone's utility increases the more assimilated his/her ethnic group, the equilibrium results are summarized in Proposition 3 in Appendix B. It is easy to see that the results are qualitatively the same since the trade off between the marginal gain from the white's social network and the cost to assimilate determines which type of equilibrium prevails. There is however a major difference: there are no more multiple equilibria, i.e. for the same parameter values, only a unique equilibrium prevails. In words, for the same observables, individuals do not choose "oppositional" identities. This is due to the specification of (4) because there is a tension between own $\left(x_{i}\right)$ and group choice $\left(\bar{x}_{N W}\right)$ of identity since an increase in $x_{i}$ increases utility while an increase in $\bar{x}_{N W}$ reduces utility. To be more precise, there are no more multiple equilibria because $x_{i}$ and $\bar{x}_{N W}$ are now strategic substitute $\left(\partial^{2} V_{N W j} / \partial x_{i} \partial \bar{x}_{N W}<0\right)$. Vives (1999) and Ballester et al. (2005) discuss the relationship between strategic complementarity/substituability and multiple/unique equilibrium.

More generally, our model shows that, in equilibrium, whites and those who choose $x_{N W}^{*}=1$ are in general respectively the most and the least favored group in terms of labor market outcomes. Indeed, whites are not discriminated against and thus benefit from a good social network. To the contrary, those who choose $x_{N W}^{*}=$ 1 have a poor social network (in particular because they do not like to interact with whites) and are discriminated against. Therefore, they have the worst labor market outcomes because unemployment is rampant and peer pressure (to conform to the community's norms and accept adverse racial preferences) has negative effects on those who are sensitive to it. These results are partly based on the fact that 
information about jobs can only be acquired through social networks (employed friends). In this respect, these individuals are totally isolated from jobs and thus have little information on job opportunities. The situation is different for those who choose $x_{N W}^{*}=0$ since they are less isolated from jobs because they have contacts with whites.

Of course, we cannot rank (expected) utilities since, for example, individuals who experience high unemployment rates can be quite "happy" since they do not interact very much with whites. The basic message here is that those who totally reject the white's culture "pay" in some sense the price of this behavior by experiencing high unemployment rates and a low probability to find a job compared to the other ethnic minorities who are more willing to adopt the white's norm. However, this does not imply that they are worse off. To see this, let us compare individuals with oppositional identities, i.e. those who choose $x_{N W}^{*}=0$ and $x_{N W}^{*}=1$. Using (17), we have:

$$
E V_{N W}(0) \gtreqless E V_{N W}(1) \Leftrightarrow \frac{e}{y_{E}-y_{U}} \lesseqgtr u(1)-u(0)
$$

since $u(1)>u(0)$. For example, when $e /\left(y_{E}-y_{U}\right)>u^{\prime}(1)$, Proposition 2 shows that both equilibria $\left(x_{N W}^{*}=0\right.$ and $\left.x_{N W}^{*}=1\right)$ occur and we cannot Pareto-rank them. If, for example, $u^{\prime}(1)<e /\left(y_{E}-y_{U}\right)<u(1)-u(0)$, those who completely assimilate to the whites $\left(x_{N W}^{*}=0\right)$ will be better off whereas if $e /\left(y_{E}-y_{U}\right)>$ $\max \left\{u^{\prime}(1), u(1)-u(0)\right\}$, then those who totally reject the white's norm $\left(x_{N W}^{*}=1\right)$ will be happier.

This model has also some interesting implications in terms family and peer pressures as well as welfare policies. In particular, there is an interesting externality generated by a non-white choosing to locate closer to the white norm. By doing so, he/she not only enjoys a higher probability of employment for him/herself, but he/she establishes a link between the white and non-white job networks. This has no effect on the rate of employment among whites, but will positively influence the probability of employment among non-whites. For the usual reasons, therefore, adoption of white identities will be underprovided. The model also suggests that, other things being equal, government guaranteed jobs (or income) should generate higher variance in identity choices. This is a particularly provocative result in light of the political debate concerning the possibility of "cultures" of aid dependence. Indeed, exogenous increases (decreases) in unemployment insurance should be associated with increases (decreases) in oppositional identity choices among minorities because there is less incentive to interact with whites. In particular, if the unemployment benefit $y_{U}$ is very low, then there is no equilibrium in which all nonwhites totally reject the white's norm. If, on the contrary $y_{U}$ is very large, then this possibility is much more likely to arise. 


\section{Conclusion}

Social unrest in Northern cities in England in 2001, September the 11th and the July 2005 London bombings have all placed the issue of ethnic identity and the notion of "Britishness" at the forefront of political debate in the UK with equivalent debates occurring in a number of other European countries (see Buruma, 2004 and Telvick, 2005). An important theme that emerges is the idea that some individuals are seen to reject the dominant society's values and that there may be a tradeoff between identification with one's own ethnic or religious group and social and economic advance. Though such frameworks have been utilized to explain racial differences in school performance in the US (Ogbu, 1978; Fordham and Ogbu, 1986; Ainsworth-Darnell and Downey, 1998) the relationship between oppositional identities and the labor market remains relatively unexplored. Apart from a few notable studies (Austen-Smith and Fryer, 2005) most of what is written on the subject seems at best a plead for further research (Heath, 2001).

This paper examines at a theoretical level the relationship between oppositional identities amongst ethnic groups and employment in the labor market. In our theoretical model ethnic preferences are predicted to reduce labor market success where preferences are gauged in terms of remoteness or otherwise to white norms. Nonwhite individuals in our model are defined with respect to their social environment and their attachments to their culture of origin and jobs are mainly found through social networks. Contingent on the strength of peer pressures, non-whites choose to adopt "oppositional" identities since some individuals may identify with the dominant culture and others may reject that culture, even if it implies unfavorable labor market outcomes. Empirical research on this issue remains limited and this we pursue in future research.

\section{References}

[1] Ainsworth-Darnell, J.W. and D.B. Downey (1998), "Assessing the oppositional culture explanation for racial/ethnic differences in school performance," American Sociological Review, 63, 536-553.

[2] Akerlof, G.A. (1980), "A theory of social custom of which unemployment may be one consequence," Quarterly Journal of Economics, 94, 749-775.

[3] Akerlof, G.A. (1997), "Social distance and social decisions", Econometrica, 65, 1005-1027.

[4] Akerlof, G.A. and R.E. Kranton (2000), "Economics and identity", Quarterly Journal of Economics, 115, 715-753. 
[5] Akerlof, G.A. and R.E. Kranton (2002), "Identity and schooling: Some lessons for the economics of education", Journal of Economic Literature, 40, 1167-1201.

[6] Alesina, A. and E. La Ferrara (2002), "Who trusts others?", Journal of Public Economics, 85, 207-234.

[7] Austen-Smith, D. and R.D. Fryer, Jr (2005), "An economic analysis of 'acting white'," Quarterly Journal of Economics, 120, 551-583.

[8] Ballester, C., Calvó-Armengol, A. and Y. Zenou (2005), "Who's who in networks. Wanted: The key player," CEPR Discussion Paper.

[9] Becker, G.S. (1957), The Economics of Discrimination, Chicago: Chicago University Press.

[10] Berman, E. (2000), "Sect, subsidy, and sacrifice: An economist's view of ultraorthodox jews", Quarterly Journal of Economics, 115, 905-953.

[11] Bernheim, B.D. (1994), "A theory of conformity," Journal of Political Economy, 102, 841-877.

[12] Buruma, I. (2004) "Letter from Amsterdam", The New Yorker, December 27.

[13] Bisin, A., Topa, G. and T. Verdier (2004), "Religious intermarriage and socialization in the United States," Journal of Political Economy, 112, 615-664.

[14] Bledsoe, T., Welch, S., Sigelman, L. and M. Combs (1995), "Residential context and racial solidarity among African Americans", American Journal of Political Science, 39, 434-458.

[15] Borjas, G.J. (1994), "The economics of immigration", Journal of Economic Literature, 32, 1667-1717.

[16] Building Cohesive Communities (2001), A Report of the Ministerial Group on Public Order and Community Cohesion.

[17] Calvó-Armengol, A. (2004), "Job contact networks", Journal of Economic Theory, 115, 191-206.

[18] Calvó-Armengol, A. and M.O. Jackson (2004), "The effects of social networks on employment and inequality," American Economic Review, 94, 426-454.

[19] Calvó-Armengol, A. and Y. Zenou (2005), "Job matching, social network and word-of-mouth communication", Journal of Urban Economics, 57, 500-522.

[20] Cantle Report (2001), Community Cohesion, Home Office. 
[21] Chiswick, B. R. (1978), "The effect of Americanization on earnings of foreign born men", Journal of Political Economy, 86, 897-921.

[22] CRE (2002), Report on Racial Segregation in the North of England.

[23] Cutler, D. M. and E. L. Glaeser (1997), "Are ghettos good or bad?", Quarterly Journal of Economics, 112, 827-872.

[24] Diamond, P. (1981), "Mobility costs, frictional unemployment, and efficiency", Journal of Political Economy, 89, 798-812.

[25] Delpit, L. (1995), Other People's Children: Cultural Conflict in the Classroom, New York: The Free Press.

[26] Dustmann, C. and F. Fabbri (2003), "Language proficiency and labour market performance of immigrants in the UK", Economic Journal, 113, 695-717.

[27] Fershtman, C. and Y. Weiss (1998), "Social rewards, externalities and stable preferences", Journal of Public Economics, 70, 53-73.

[28] Fordham S. and J.U. Ogbu (1986), "Black student'school success: Coping with the burden of acting White", The Urban Review, 18, 176-206.

[29] Heath, A. (2001), Ethnic Minorities in the Labour Market, Report to the PIU, Cabinet office, October 2001.

[30] Iannaccone, L.R. (1998), "Introduction to the economics of religion", Journal of Economic Literature, 36, 1465-1496.

[31] Ihlanfeldt, K. and B. Scafidi (2002), "Black self-segregation as a cause of housing segregation. Evidence from the multi-city study of urban inequality", Journal of Urban Economics, 51, 366-390.

[32] Kandel, E. and E.P. Lazear (1992), "Peer pressure and partnerships," Journal of Political Economy, 100, 801-817.

[33] Lazear, E.P. (1999), "Culture and language", Journal of Political Economy, 107, S95-S126.

[34] McManus, W.S., Gould, W. and F. Welch (1983), "Earnings of hispanic men: The role of English language proficiency", Journal of Labor Economics, 1, 101130.

[35] Modood, T. et al. (1997), Ethnic Minorities in Britain: Diversity and Disadvantage, London: Policy Studies Institute.

[36] Montgomery, J. (1991), "Social networks and labor-market outcomes: Toward an economic analysis", American Economic Review, 81, 1408-1418. 
[37] Mortensen, D.T. and T. Vishwanath (1994), "Personal contacts and earnings. It is who you know!", Labour Economics, 1, 187-201.

[38] Ogbu, J.U. (1997), "Beyond language: ebonics, proper English and identity in a Black American speech community", Unpublished manuscript, University of California at Berkeley, Department of Anthropology.

[39] Ogbu, J.U. (1978), Minority Education and Caste, New York: Academic Press.

[40] Pagnini, D.L. and S.P. Morgan (1990), "Intermarriage and social distance among U.S. immigrants at the turn of the century", American Journal of Sociology, 96, 405-432.

[41] Patacchini, E. and Y. Zenou (2005), "Crime and conformism", CEPR Discussion Paper.

[42] Peach, C. (1996) "Introduction," in C. Peach (ed.), The Ethnic Minority Populations of Britain, London: HMSO.

[43] Peach, C. (2005) "Social integration and social mobility: spatial segregation and intermarriage of the Caribbean population in Britain" in Loury, G.C., Modood, T. and Teles, S.M.(editors) Ethnicity, Social Mobility, and Public Policy, Cambridge: Cambridge University Press.

[44] Pissarides, C.A. (2000), Equilibrium Unemployment Theory, 2nd edition, Cambridge: MIT Press.

[45] Selod, H. and Y. Zenou (2005), "Does city structure affect the labor market outcomes of black workers?", Economic Journal, forthcoming.

[46] Telvick, M. (2005) "Identity crisis: old Europe meets new Islam", Frontline, January 25, available at http://www.pbs.org.

[47] Topa, G. (2001), "Social interactions, local spillovers and unemployment", Review of Economic Studies, 68, 261-295.

[48] Vives, X. (1999), Oligopoly Pricing: Old Ideas and New Tools, Cambridge: MIT Press. 


\section{APPENDIX A: Proof of the propositions}

Lemma 1 Whatever the location $0 \leq x \leq 1$ chosen by non-whites, the unemployment rate of whites is always lower than that of non-whites, i.e.

$$
u_{W}<u_{N W}
$$

Proof. This is obvious since all whites are located in $x=0$ and they are not discriminated against. So even if non-whites choose the "best" location in terms of labor market outcomes, i.e. $x=0$, they will still experience a higher unemployment rate because of labor discrimination.

Lemma 2 Assume $\mu>\lambda$. Then

(i) The unemployment rate of whites is uniquely determined, strictly positive, strictly between 0 and 1 and is given by:

$$
u_{W}=\frac{\delta+\mu+\lambda N_{W}-\sqrt{\left(\delta+\mu+\lambda N_{W}\right)^{2}-4 \lambda N_{W} \delta}}{2 \lambda N_{W}}
$$

(ii) For non-whites, we have:

(iia) When $x=0$, the unemployment rate of non-whites is uniquely determined, strictly positive, strictly between 0 and 1 and is given by:

$$
u(0) \equiv u_{N W}(0)=\frac{\delta}{\delta+\mu m+\lambda m\left(1-u_{W}\right) N_{W}}
$$

(iib) When $x=1$, the unemployment rate of non-whites is uniquely determined, strictly positive, strictly between 0 and 1 and is equal to:

$$
u(1) \equiv u_{N W}(1)=\frac{\delta+\left(\mu+\lambda N_{W}\right) m-\sqrt{\left[\delta+\left(\mu+\lambda N_{W}\right) m\right]^{2}-4 \delta \lambda N_{W} m}}{2 \lambda N_{W} m}
$$

(iic) When $0<x<1$, the unemployment rate of non-whites is uniquely determined, strictly positive, strictly between 0 and 1 and is given by:

$$
u_{N W}(x)=\frac{\delta+\mu m+\lambda m\left[(1-x)\left(1-u_{W}\right) N_{W}+x N_{N W}\right]-\sqrt{\Delta}}{2 \lambda N_{N W} m x}
$$

where

$$
\Delta=\left[\delta+\mu m+\lambda m\left[(1-x)\left(1-u_{W}\right) N_{W}+x N_{N W}\right]\right]^{2}-4 \delta N_{N W} \lambda m x>0
$$


Proof. ( $i$ ) The unemployment rate of whites is defined by (14), which is equivalent to:

$$
\lambda N_{W} u_{W}^{2}-\left(\delta+\mu+\lambda N_{W}\right) u_{W}+\delta=0
$$

The discriminant is $\Delta_{W}=\left(\delta+\mu+\lambda N_{W}\right)^{2}-4 \lambda N_{W} \delta$. If $\mu>\lambda$, then $\Delta_{W}>0$. We thus have two distinct roots that are given by

$$
u_{W}=\frac{\delta+\mu+\lambda N_{W} \pm \sqrt{\left(\delta+\mu+\lambda N_{W}\right)^{2}-4 \lambda N_{W} \delta}}{2 \lambda N_{W}}
$$

and both of them are strictly positive. Let us show that the root with the highest value is strictly greater than 1 . This is equivalent to

$$
\delta+\mu+\sqrt{\left(\delta+\mu+\lambda N_{W}\right)^{2}-4 \lambda N_{W} \delta}>\lambda N_{W}
$$

which is always true as soon as $\mu>\lambda$ (since $N_{W}<1$ ). Let us show that the root with the lowest value is strictly less than 1 . This is equivalent to

$$
\delta+\mu-\lambda N_{W}<\sqrt{\left(\delta+\mu+\lambda N_{W}\right)^{2}-4 \lambda N_{W} \delta}
$$

or

$$
\begin{gathered}
4 \lambda N_{W} \delta<\left(\delta+\mu+\lambda N_{W}\right)^{2}-\left(\delta+\mu-\lambda N_{W}\right)^{2} \\
\Leftrightarrow \mu>0
\end{gathered}
$$

We have thus shown that there is a unique $u_{W}$ such that $0<u_{W}<1$ and it is given by (18).

Let us now focus on $u_{N W}$, the unemployment rate of nonwhites, which is defined by (15). Different cases must be considered.

(iia) When $x=0,(15)$ reduces to

$$
\left[\delta+\mu m+\lambda m\left(1-u_{W}\right) N_{W}\right] u_{N W}(0)-\delta=0
$$

By solving these equations, we obtain

$$
u_{N W}(0)=\frac{\delta}{\delta+\mu m+\lambda m\left(1-u_{W}\right) N_{W}}>0
$$

It is obvious that $u_{N W}(0)$ is less than 1 since $\delta<\delta+\mu m+\lambda m\left(1-u_{W}\right) N_{W}$.

We have thus shown that, when $x=0$, there is a unique $u_{N W}$ such that $0<$ $u_{N W}(0)<1$ and it is given by $(19)$.

(iib) When $x=1,(15)$ reduce to

$$
\lambda N_{N W} m u_{N W}^{2}(1)-\left[\delta+\left(\mu+\lambda N_{N W}\right) m\right] u_{N W}(1)+\delta=0
$$

The discriminant is given by $\Delta_{N W}(1)=\left[\delta+\left(\mu+\lambda N_{N W}\right) m\right]^{2}-4 \lambda N_{W} m \delta$. It is easy to verify that if $\mu>\lambda$, then $\Delta_{N W}>0$. We thus have two distinct roots that are given by

$$
u_{N W}(1)=\frac{\delta+\left(\mu+\lambda N_{W}\right) m \pm \sqrt{\left[\delta+\left(\mu+\lambda N_{W}\right) m\right]^{2}-4 \lambda m \delta N_{W}}}{2 \lambda N_{W} m}
$$


and both of them are strictly positive. Let us show that the root with the highest value is strictly greater than 1 . This is equivalent to

$$
\delta+\left(\mu+\lambda N_{W}\right) m+\sqrt{\left[\delta+\left(\mu+\lambda N_{W}\right) m\right]^{2}-4 \lambda N_{W} m \delta}>2 \lambda N_{W} m
$$

which is always true as soon as $\mu>\lambda$. Let us show that the root with the lowest value is strictly less than 1 . This is equivalent to

$$
\delta+\left(\mu+\lambda N_{W}\right) m-\sqrt{\left[\delta+\left(\mu+\lambda N_{W}\right) m\right]^{2}-4 \lambda N_{W} m \delta}<2 \lambda N_{W} m
$$

or

$$
\begin{gathered}
\left(\delta+\mu m-\lambda N_{W} m\right)^{2}<\left[\delta+\left(\mu+\lambda N_{W}\right) m\right]^{2}-4 \lambda N_{W} m \delta \\
\Leftrightarrow \mu m>0
\end{gathered}
$$

We have thus shown that, when $x=1$, there is a unique $u_{N W}$ such that $0<$ $u_{N W}(1)<1$ and it is given by $(20)$.

(iic) When $0<x<1$, (15) reduces to:

$$
\lambda N_{N W} m x u_{N W}^{2}-\left[\delta+\mu m+\lambda m\left[(1-x)\left(1-u_{W}\right) N_{W}+x N_{N W}\right]\right] u_{N W}+\delta=0
$$

The discriminant of this equation is given by:

$$
\Delta=\left[\delta+\mu m+\lambda m\left[(1-x)\left(1-u_{W}\right) N_{W}+x N_{N W}\right]\right]^{2}-4 \delta N_{N W} \lambda m x
$$

Let us check that it is positive. This is equivalent to:

$$
\left[\delta+\mu m+\lambda m\left[(1-x)\left(1-u_{W}\right) N_{W}+x N_{N W}\right]\right]^{2}>4 \delta \lambda N_{N W} m x
$$

or

$$
\begin{gathered}
(\delta+\mu m)^{2}+\lambda^{2} m^{2}\left[(1-x)\left(1-u_{W}\right) N_{W}+x N_{N W}\right]^{2} \\
+2(\delta+\mu m) \lambda m\left[(1-x)\left(1-u_{W}\right) N_{W}+x N_{N W}\right]>4 \delta \lambda N_{N W} m x
\end{gathered}
$$

or

$$
\begin{gathered}
\delta^{2}+\mu^{2} m^{2}+2 \delta \mu m+\lambda^{2} m^{2}\left[(1-x)\left(1-u_{W}\right) N_{W}+x N_{N W}\right]^{2} \\
+2 \mu \lambda m^{2}\left[(1-x)\left(1-u_{W}\right) N_{W}+x N_{N W}\right]+2 \delta \lambda m\left[(1-x)\left(1-u_{W}\right) N_{W}\right]>2 \delta \lambda N_{N W} m x \\
\Leftrightarrow \delta^{2}+\mu^{2} m^{2}+\lambda^{2} m^{2}\left[(1-x)\left(1-u_{W}\right) N_{W}+x N_{N W}\right]^{2} \\
+2 \mu \lambda m^{2}\left[(1-x)\left(1-u_{W}\right) N_{W}+x N_{N W}\right]+2 \delta \lambda m\left[(1-x)\left(1-u_{W}\right) N_{W}\right] \\
+2 \delta m\left(\mu-\lambda N_{N W} x\right)>0
\end{gathered}
$$

This last inequality is always true since $\mu>\lambda N_{N W} x$ because $N_{N W}<1, x<1$ and $\mu>\lambda$. Thus $\Delta>0$. As a result, we have two distinct roots that are given by:

$$
u_{N W}(x)=\frac{\delta+\mu m+\lambda m\left[(1-x)\left(1-u_{W}\right) N_{W}+x N_{N W}\right] \pm \sqrt{\Delta}}{2 \lambda N_{N W} m x}
$$


and both of them are strictly positive. Let us show that the root with the highest value is strictly greater than 1 . This is equivalent to

$$
\delta+\mu m+\lambda m\left[(1-x)\left(1-u_{W}\right) N_{W}+x N_{N W}\right]+\sqrt{\Delta}>2 \lambda N_{N W} m x
$$

or

$$
\delta+m\left(\mu-\lambda N_{N W} x\right)+\lambda m\left[(1-x)\left(1-u_{W}\right) N_{W}\right]+\sqrt{\Delta}>0
$$

which is always true because $N_{N W}<1, x<1$ and $\mu>\lambda$. Let us now show that the root with the lowest value is strictly less than 1 . We have:

$$
\delta+\mu m+\lambda m\left[(1-x)\left(1-u_{W}\right) N_{W}+x N_{N W}\right]-\sqrt{\Delta}<2 \lambda N_{N W} m x
$$

which, using the value of $\Delta$, is equivalent to (taking the square on both sides):

$$
\lambda N_{N W} m x-\delta+\sqrt{\Delta}>0
$$

Then, taking again the square on both sides gives and using the value of $\Delta$ :

$\left[\delta+\mu m+\lambda m\left[(1-x)\left(1-u_{W}\right) N_{W}+x N_{N W}\right]\right]^{2}>\delta^{2}+\lambda^{2} N_{N W}^{2} m^{2} x^{2}+2 \delta \lambda N_{N W} m x$ which is equivalent to:

$$
\left[\delta+\mu m+\lambda m\left[(1-x)\left(1-u_{W}\right) N_{W}+x N_{N W}\right]\right]^{2}>\left(\delta+\lambda N_{N W} m x\right)^{2}
$$

or

$$
m\left(\mu-\lambda N_{N W} x\right)+\lambda m\left[(1-x)\left(1-u_{W}\right) N_{W}+x N_{N W}\right]>0
$$

This last inequality is again always true because $N_{N W}<1, x<1$ and $\mu>\lambda$.

We have thus shown that, when $0<x<1$, there is a unique $u_{N W}(x)$ such that $0<u_{N W}(x)<1$ and it is given by $(21)$.

\section{Proof of Proposition 2}

Before proving this proposition, we need the following three Lemmata.

Lemma 3 Assume $\mu>\lambda$. The function $u_{N W}(x)$ is strictly increasing with $x$ on the interval $[u(0), u(1)]$, where $0<u(0)<1$ and $0<u(1)<1$ are respectively defined by (19) and (20). More precisely, we have:

$$
\frac{\partial u_{N W}(x)}{\partial x} \equiv u_{N W}^{\prime}(x)=\frac{\lambda m u_{N W}(x)\left[\left(1-u_{W}\right) N_{W}-\left(1-u_{N W}(x)\right) N_{N W}\right]}{D(x)}>0
$$

where $D(x) \equiv \delta+\mu m+\lambda m\left[(1-x)\left(1-u_{W}\right) N_{W}+x N_{N W}\right]-2 \lambda N_{N W} m x u_{N W}(x)$. 
Proof. By totally differentiating (23), we obtain (24). Furthermore, using Lemma 1 and the fact that $N_{W}>N_{N W}$, the numerator of (24) is clearly strictly positive. Finally, the denominator $D(x)$ of $(24)$ can be rewritten as

$D(x)=\delta+m\left(\mu-\lambda N_{N W} x u_{N W}(x)\right)+\lambda m(1-x)\left(1-u_{W}\right) N_{W}+\lambda N_{N W} m x\left(1-u_{N W}(x)\right)$

Since $\mu>\lambda$, and $x, N_{N W}$ and $u_{N W}$ are all less than 1 , then $m\left(\mu-\lambda N_{N W} x u_{N W}(x)\right)>$ 0 . As a result, the denominator $D(x)$ is strictly positive and $\partial u_{N W}(x) / \partial x>0$.

Lemma 4 Assume $\mu>\lambda$. Then, the function $u_{N W}^{\prime}(x)$ is strictly increasing and convex on the interval $\left[u^{\prime}(0), u^{\prime}(1)\right]$, where $u^{\prime}(0)$ and $u^{\prime}(1)$ have both finite values and are respectively given by

$$
\begin{aligned}
& u^{\prime}(0) \equiv u_{N W}^{\prime}(0)=\frac{\lambda m u(0)\left[\left(1-u_{W}\right) N_{W}-(1-u(0)) N_{N W}\right]}{\delta+\mu m+\lambda N_{W} m\left(1-u_{W}\right)}>0 \\
& u^{\prime}(1) \equiv u_{N W}^{\prime}(1)=\frac{\lambda m u(1)\left[\left(1-u_{W}\right) N_{W}-(1-u(1)) N_{N W}\right]}{\delta+\mu m+\lambda N_{N W} m(1-2 u(1))}>0
\end{aligned}
$$

where $u_{W}, u(0)$ and $u(1)$ are respectively defined by (18), (19) and (20).

Proof. By differentiating (24) with respect to $x$, we obtain:

$$
\begin{gathered}
\frac{\partial^{2} u_{N W}(x)}{\partial x^{2}} \equiv u_{N W}^{\prime \prime}(x) \\
=\frac{\lambda m u^{\prime} A D(x)+\lambda^{2} m^{2} u_{N W}(x)\left[\left(1-u_{W}\right) N_{W}-\left(1-u_{N W}(x)\right) N_{N W}\right]\left(A+2 N_{N W} x u^{\prime}\right)}{D(x)^{2}}
\end{gathered}
$$

where $u^{\prime} \equiv u_{N W}^{\prime}(x)$ and

$$
A \equiv\left[\left(1-u_{W}\right) N_{W}-\left(1-2 u_{N W}(x)\right) N_{N W}\right]>0
$$

which is clearly strictly positive using Lemma 1 and the fact that $N_{W}>N_{N W}$. As a result, since $D(x)>0$ and $u^{\prime}>0$ by Lemma 3 , then $u_{N W}^{\prime \prime}(x)>0$.

This shows that $u_{N W}^{\prime}(x)$ is strictly increasing. To calculate the values of $u^{\prime}(0)$ and $u^{\prime}(1)$, it remains to respectively plug the value $x=0$ and $x=1$ in (24) and we easily obtain (25) and (26). Finally, let us show that $u^{\prime}(0)$ and $u^{\prime}(1)$ have both finite values. Since $u^{\prime}(0)<u^{\prime}(1)$, it suffices to show that $u^{\prime}(1)$ is bounded above. In fact, it is easy to see that $u^{\prime}(1)<1 /\left[(1-u(1)) N_{N W}\right]$. Indeed, this rewrites

$$
\frac{\lambda m u(1)\left[\left(1-u_{W}\right) N_{W}-(1-u(1)) N_{N W}\right]}{\delta+\mu m+\lambda N_{N W} m(1-2 u(1))}<\frac{1}{(1-u(1)) N_{N W}}
$$

or equivalently

$$
\lambda m u(1)\left(1-u_{W}\right) N_{W}<\frac{\delta+\mu m-\lambda N_{N W} m u(1)}{(1-u(1)) N_{N W}}+\lambda m+\lambda m u(1)(1-u(1)) N_{N W}
$$


Since $\mu>\lambda$ implies that $\mu m-\lambda N_{N W} m u(1)$, it suffices to show that

$$
\lambda m u(1)\left(1-u_{W}\right) N_{W}<\lambda m
$$

which is always true because $u(1)\left(1-u_{W}\right) N_{W}<1$. This implies that $u^{\prime}(1)$ has a finite value and thus both $u^{\prime}(0)$ and $u^{\prime}(1)$ have finite values.

Let us now show that the function $u_{N W}^{\prime}(x)$ is concave on the interval $\left[u^{\prime}(0), u^{\prime}(1)\right]$. By differentiating $u_{N W}^{\prime \prime}(x)$, we obtain:

$$
\begin{gathered}
\lambda m D^{3} u_{N W}^{\prime \prime \prime}(x) \\
=\lambda m D\left[u_{N W}^{\prime}\left[\left(1-u_{W}\right) N_{W}-\left(1-u_{N W}\right) N_{N W}\right]+u_{N W} u_{N W}^{\prime} N_{N W}\right]\left(A+2 N_{N W} x u_{N W}^{\prime}\right) \\
+\lambda m D u_{N W}\left[\left(1-u_{W}\right) N_{W}-\left(1-u_{N W}\right) N_{N W}\right]\left(A^{\prime}+2 N_{N W}\left(u_{N W}^{\prime}+x u_{N W}^{\prime \prime}\right)\right) \\
-2 \lambda m\left[\lambda m u_{N W}\left[\left(1-u_{W}\right) N_{W}-\left(1-u_{N W}\right) N_{N W}\right]\left(A+2 N_{N W} x u_{N W}^{\prime}\right)\right] D^{\prime}
\end{gathered}
$$

where $D \equiv D(x)$ is defined in Lemma 3. By denoting by $Z \equiv\left(1-u_{W}\right) N_{W}-$ $\left(1-u_{N W}\right) N_{N W}>0$, this is equivalent to:

$$
\begin{gathered}
\lambda m D^{3} u_{N W}^{\prime \prime \prime}(x)=\lambda m D\left[u^{\prime} Z+u u^{\prime} N_{N W}\right]\left(A+2 N_{N W} x u^{\prime}\right)+2 N_{N W} x u^{\prime \prime} \lambda m D u_{N W} Z \\
+\lambda m u_{N W} Z\left[D A^{\prime}-2 \lambda m A+2 N_{N W} u^{\prime} D-4 N_{N W} u^{\prime} \lambda m x D^{\prime}\right]
\end{gathered}
$$

The first two terms are positive, thus let us show that the last term $D A^{\prime}-2 \lambda m A+$ $2 N_{N W} u^{\prime} D-4 N_{N W} u^{\prime} \lambda m x D^{\prime}$ is positive. Observe that

$$
D^{\prime}=-2 m \lambda N_{N W} x u_{N W}^{\prime}(x)-m \lambda\left[\left(1-u_{W}\right) N_{W}-N_{N W}\right]<0
$$

and thus all terms are positive but $-2 \lambda m A<0$. Since we have:

$$
\begin{gathered}
2 \lambda m A=2 \lambda m\left[\left(1-u_{W}\right) N_{W}-\left(1-2 u_{N W}(x)\right) N_{N W}\right] \\
D A^{\prime}+2 N_{N W} u^{\prime} D=4 N_{N W} \lambda m u_{N W}\left[\left(1-u_{W}\right) N_{W}-\left(1-u_{N W}\right) N_{N W}\right] \\
-4 N_{N W} u^{\prime} \lambda m x D^{\prime}=4 N_{N W} u^{\prime} \lambda m x\left[2 m \lambda N_{N W} x u_{N W}^{\prime}(x)+m \lambda\left[\left(1-u_{W}\right) N_{W}-N_{N W}\right]\right] \\
=8\left(N_{N W}\right)^{2}\left(u^{\prime}\right)^{2} \lambda^{2} m^{2} x^{2}+4 N_{N W} u^{\prime} \lambda^{2} m^{2} x\left[\left(1-u_{W}\right) N_{W}-N_{N W}\right]
\end{gathered}
$$

it is easy to show that, in the interval $\left[u^{\prime}(0), u^{\prime}(1)\right], D A^{\prime}-2 \lambda m A+2 N_{N W} u^{\prime} D-$ $4 N_{N W} u^{\prime} \lambda m x D^{\prime}$ is strictly positive and thus $u_{N W}^{\prime \prime \prime}(x)>0$.

Lemma 5 The expected utility function $E V_{N W}\left(x_{i}\right)$ is strictly concave on $[0,1]$. 
Proof. The expected utility function $E V_{N W}\left(x_{i}\right)$ is given by:

$$
E V_{N W}\left(x_{i}\right)=y_{E}+e x_{i} \bar{x}_{N W}-u_{N W}\left(x_{i}\right)\left(y_{E}-y_{U}\right)
$$

By differentiating twice this function, we easily obtain:

$$
\frac{\partial^{2} E V_{N W}\left(x_{i}\right)}{\partial x^{2}}=-u_{N W}^{\prime \prime}\left(x_{i}\right)\left(y_{E}-y_{U}\right)<0
$$

which is strictly negative since, in Lemma 4 , we have shown that $u_{N W}^{\prime \prime}(x)$, defined by (27), is strictly positive.

\section{Let us now prove Proposition 2.}

The first order condition for non-whites is given by:

$$
\frac{\partial E V_{N W}\left(x_{i}\right)}{\partial x_{i}}=e \bar{x}_{N W}-u_{N W}^{\prime}\left(x_{i}\right)\left(y_{E}-y_{U}\right)=0
$$

We focus on symmetric equilibria. Thus $\bar{x}_{N W}=x_{i}=x_{N W}^{*}$. This first order condition can be written as

$$
e x_{N W}^{*}-u_{N W}^{\prime}\left(x_{N W}^{*}\right)\left(y_{E}-y_{U}\right)=0
$$

We have to study this equation. Let us denote $g(x) \equiv \frac{e}{y_{E}-y_{U}} x$. Then this equation can be written as :

$$
g\left(x_{N W}^{*}\right)=u_{N W}^{\prime}\left(x_{N W}^{*}\right)
$$

We know from Lemma 4 that $u_{N W}^{\prime}(x)$ is strictly increasing on the interval $\left[u^{\prime}(0), u^{\prime}(1)\right]$, where $u^{\prime}(0)$ and $u^{\prime}(1)$ are both strictly positive and have finite values. Moreover, it easy to see that $g(\cdot)$ is a line with a positive slope of $\frac{e}{y_{E}-y_{U}}$ and with $g(0)=0$ and $g(1)=\frac{e}{y_{E}-y_{U}}$.

(i) Consider first the case when $e /\left(y_{E}-y_{U}\right)<u^{\prime}(0)$. Then it is clear that $u_{N W}^{\prime}\left(x_{N W}\right)>g\left(x_{N W}\right), \forall x_{N W} \in[0,1]$ and thus $\partial E V_{N W}(x) / \partial x<0$. As a result, the only possible equilibrium is when all workers choose $x_{N W}^{*}=0$. It is obvious that no other equilibrium can exist since, in this case, worker $i$ will always deviate to choose $x_{N W}^{*}=0$ (see Figure 1). In this case, the unemployment rate of all non-whites is $u(0) \equiv u_{N W}(0)$ and is given by (19).

(iii) Consider now the other extreme case when $e /\left(y_{E}-y_{U}\right)>u^{\prime}(1)$. From Proposition 1, we know that the equilibrium when all workers choose $x_{N W}^{*}=0$ exists. There is clearly another equilibrium in which all workers choose $x_{N W}^{*}=1$. Indeed, if $x_{N W}^{*}=1$, then the first order condition writes: $e-u_{N W}^{\prime}(1)\left(y_{E}-y_{U}\right)=0$. Thus if $e /\left(y_{E}-y_{U}\right) \geq u^{\prime}(1)$, we have that $\partial E V_{N W}(x) / \partial x>0, \forall x \in[0,1]$. As a result, all workers choose $x_{N W}^{*}=1$. Their unemployment rate $u(1) \equiv u_{N W}(1)$ is 
given by (20). Can we have another equilibrium in which $0<x_{N W}^{*}<1$ ? The answer is yes. Indeed, we have that $u^{\prime}(0)>0$ and that $g(1)>u^{\prime}(1)$. Since the functions $g(\cdot)$ and $u^{\prime}(\cdot)$ are both continuous and increasing in $x$, they have to intersect only once at $x$ between 0 and 1 . There is thus another equilibrium in which all workers choose a unique $0<\widehat{x}_{N W}^{*}<1$. Their unemployment rate is given by (21). Figure 4 illustrates this case.

(ii) Consider the intermediate case when $u^{\prime}(0)<e /\left(y_{E}-y_{U}\right)<u^{\prime}(1)$. From Proposition 1, we know that the equilibrium when all workers choose $x_{N W}^{*}=0$ exists. Is it possible to have an equilibrium in which all workers choose $x_{N W}^{*}=1$ ? If this is the case, the first order condition for $i$ is: $e-u^{\prime}(1)\left(y_{E}-y_{U}\right)$, which is always negative so that the best reply for $i$ is to choose $x_{N W}^{*}=0$. Thus an equilibrium in which all workers choose $x_{N W}^{*}=1$ cannot exist in this case. Consider thus symmetric equilibria in which all workers choose $0<x_{N W}^{*}<1$. Generically, two cases may then arise. Case (iia) Either the curve $u^{\prime}(x)$ is always above the line $g(x)$ (see Figure 2) $\forall x \in[0,1]$. This is true is $u^{\prime}(0)$ is large enough. In this case, the only equilibrium is that all workers choose $x_{N W}^{*}=0$. Case $(i i b)$ Or the curve $u^{\prime}(x)$ cuts the line $g(x)$ twice at $0<\underline{x}_{N W}^{*}<1$ and $0<\bar{x}_{N W}^{*}<1$ (see Figure 3). ${ }^{13}$ This is true is $u^{\prime}(0)$ is small enough. We thus have two equilibria in which in one case all workers choose $\underline{x}_{N W}^{*}$ and in the other they all choose $\bar{x}_{N W}^{*}$. It should be clear that if all workers choose for example $\underline{x}_{N W}^{*}$ then this is an equilibrium since no worker will deviate because at $\underline{x}_{N W}^{*}$ his/her expected utility is maximum. In both equilibria, their unemployment rate is given by (21) with different values when $x=\underline{x}_{N W}^{*}$ and when $x=\bar{x}_{N W}^{*}$. It is finally easy to verify that $\widehat{x}_{N W}^{*}$ defined above in case $(i i i)$ is such that $\underline{x}_{N W}^{*}<\widehat{x}_{N W}^{*}<\bar{x}_{N W}^{*}$.

Let us now show that each equilibrium is stable. Basically, the only variable that is dynamic is the unemployment rate. The equation of evolution of unemployment is given by (12), which we can be written as:

$$
\dot{u_{k}}=\delta\left(1-u_{k}\right)-m_{k} \theta_{k} u_{k} \quad, \quad k=N W, W
$$

By solving this differential equation, we easily obtain:

$$
u(t)=\exp \left\{-\left[\delta+m_{k} \theta_{k}\right] t\right\}+u_{k}^{*}
$$

where $u_{k}^{*}$ is the steady-state unemployment rate given by (13), i.e.

$$
u_{k}=\frac{\delta}{\delta+m_{k} \theta_{k}} \quad k=N W, W
$$

It is easy to see that

$$
\lim _{t \rightarrow+\infty} u(t)=u_{k}^{*}
$$

\footnotetext{
${ }^{13}$ There is also another case when the curve $u^{\prime}(x)$ cuts the line $g(x)$ only once. We ignore this case since this happens on a set of measure zero.
} 
As a result, the equation for the evolution of unemployment (12) is stable, i.e. for any given initial condition it always converges to its steady state value, and thus, for each regime, the steady-state equilibrium is also stable.

Finally, it is straightforward to see that

$$
u_{W}<u_{S S}<\underline{u}_{I N}<\widehat{u}_{I N}<\bar{u}_{I N}<u_{C O}
$$

since, by Lemma 1 , the unemployment rate of whites $u_{W}$ is always lower than any unemployment rate of nonwhites and the only difference between nonwhites' unemployment rates is the contact with whites through $1-u_{W}$. 


\section{APPENDIX B: An alternative formulation}

The utility function is given by (4), that is:

$$
V_{N W j}\left(x_{i}\right)=y_{j}+e x_{i}\left(K-\bar{x}_{N W}\right)
$$

where $K>1$, and thus

$$
E V_{N W}\left(x_{i}\right)=y_{E}+e x_{i}\left(K-\bar{x}_{N W}\right)-u_{N W}\left(x_{i}\right)\left(y_{E}-y_{U}\right)
$$

First-order condition gives

$$
\frac{\partial E V_{N W}\left(x_{i}\right)}{\partial x_{i}}=e\left(K-\bar{x}_{N W}\right)-u_{N W}^{\prime}\left(x_{i}\right)\left(y_{E}-y_{U}\right)
$$

First, observe that Proposition 1 is not true anymore so that there not always exists a steady-state equilibrium in which all nonwhite workers choose to totally adopt the white's norm, i.e. $x_{N W}^{*}=\bar{x}_{N W}^{*}=0$. Using the same proof as for Proposition 2 and observing that Lemmata 1, 2, 3, 4 and 5 are still valid and that now

$$
g(x)=\frac{e(K-x)}{y_{E}-y_{U}}
$$

we have the following result:

Proposition 3 Assume $\mu>\lambda$. If the utility is described by (4), then we have:

(i) If eK/(yE $\left.-y_{U}\right) \leq u^{\prime}(0)$, there is a unique symmetric stable steady-state equilibrium in which all nonwhite workers choose to totally adopt the white's norm, i.e. $x_{N W}^{*}=\bar{x}_{N W}^{*}=0$. Their unemployment rate $u_{S S} \equiv u(0)$ is given by (19).

(ii) If $e(K-1) /\left(y_{E}-y_{U}\right)<u^{\prime}(1)$, there is a unique symmetric stable steadystate equilibrium in which all nonwhite workers choose to partially adopt the white's norm, i.e. $0<x_{N W}^{*}=\bar{x}_{N W}^{*}<1$. Their unemployment rate $\widehat{u}_{N W} \equiv$ $u_{N W}\left(\bar{x}_{N W}^{*}\right)$ is given by (21).

(iii) If e $(K-1) /\left(y_{E}-y_{U}\right) \geq u^{\prime}(1)$, there is a unique symmetric stable steady-state equilibrium in which all nonwhite workers choose to totally reject the white's norm, i.e. $x_{N W}^{*}=\bar{x}_{N W}^{*}=1$. Their unemployment rate $u_{C O} \equiv u(1)$ is given by (20).

The unemployment rate of whites $u_{W}$ is given by (18). We also have that:

$$
u_{W}<u_{S S}<\widehat{u}_{N W}<u_{C O}
$$



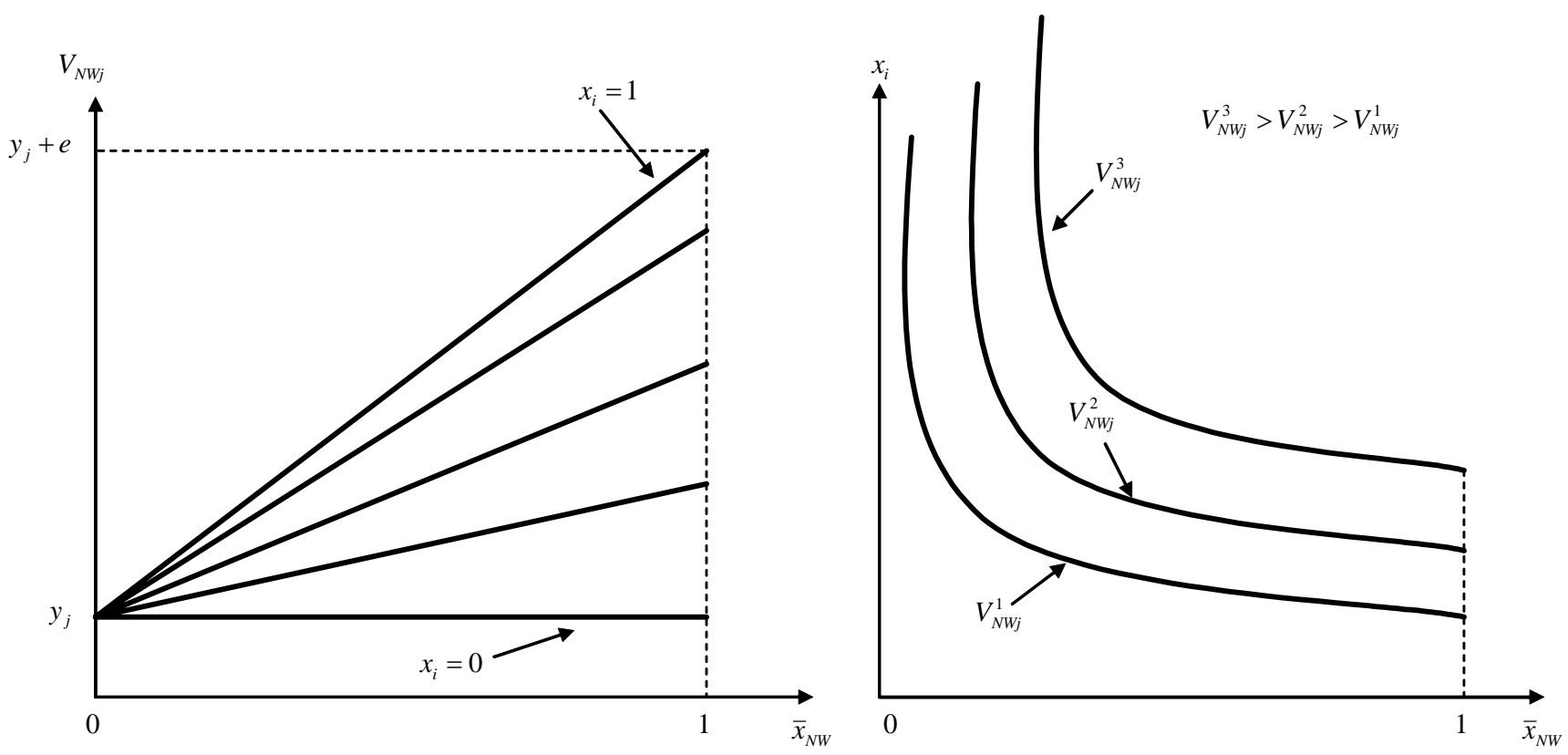

Figure 1: Utility and group externalities for $V_{N M j}=y_{j}+e x_{i} \bar{x}_{N W}$ 


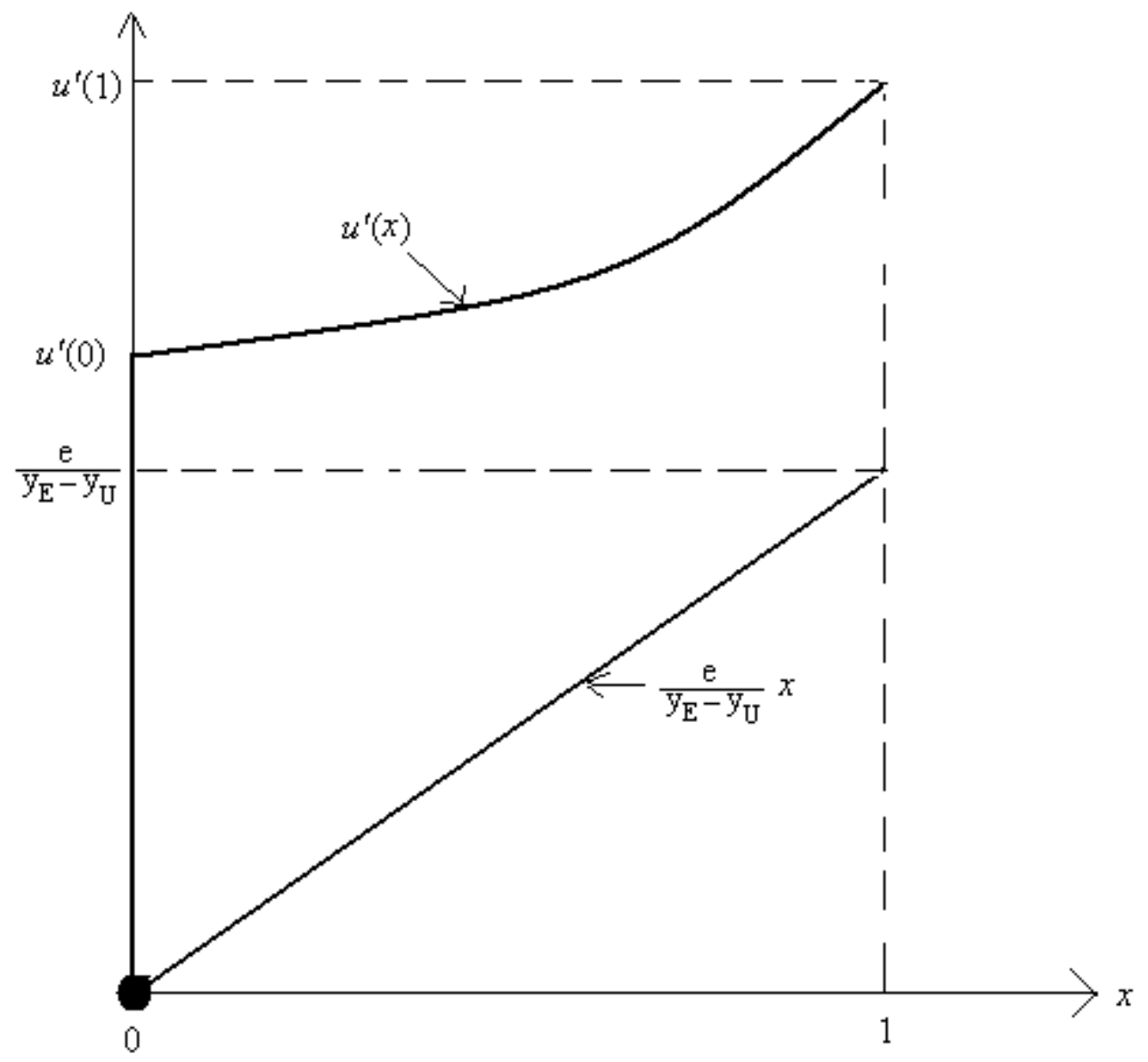

Figure 2a: Case $(i)$ when $e /\left(y_{E}-y_{U}\right)<u^{\prime}(0)$ 


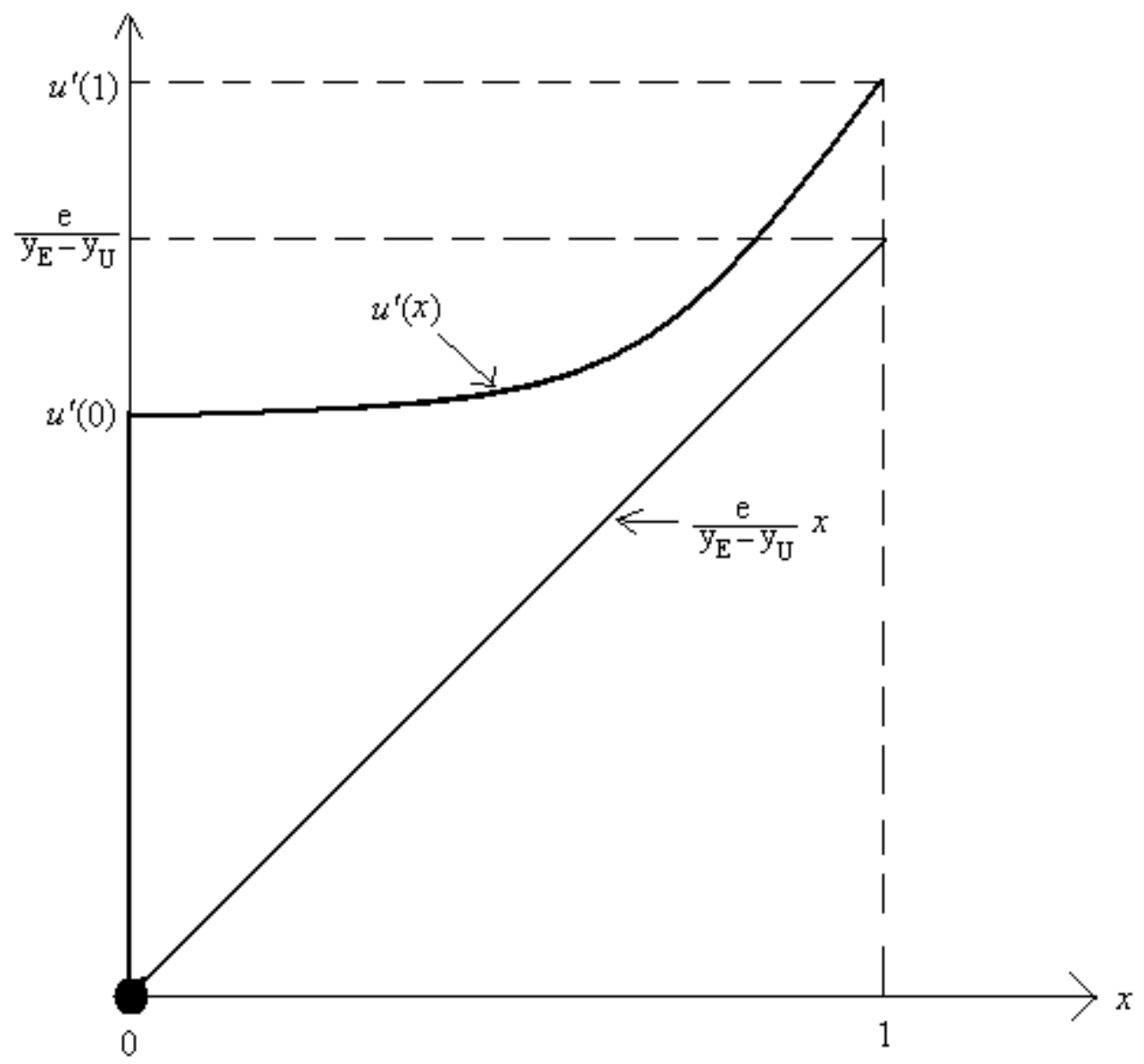

Figure 2b: Case $($ iia $)$ when $u^{\prime}(0)<e /\left(y_{E}-y_{U}\right)<u^{\prime}(1)$ and $u^{\prime}(0)$ is large enough 


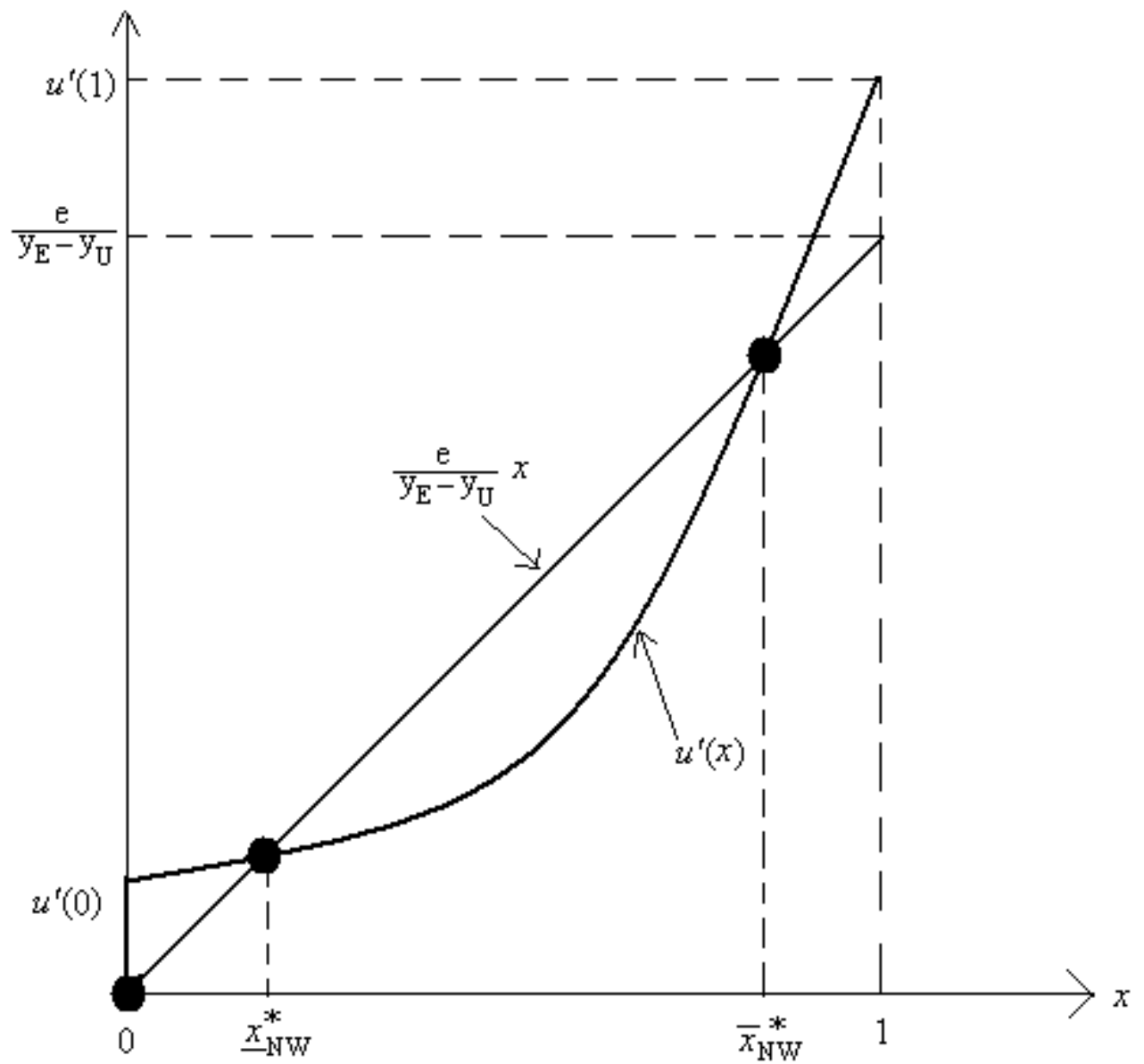

Figure 2c: Case $(i i b)$ when $u^{\prime}(0)<e /\left(y_{E}-y_{U}\right)<u^{\prime}(1)$ and $u^{\prime}(0)$ is small enough 


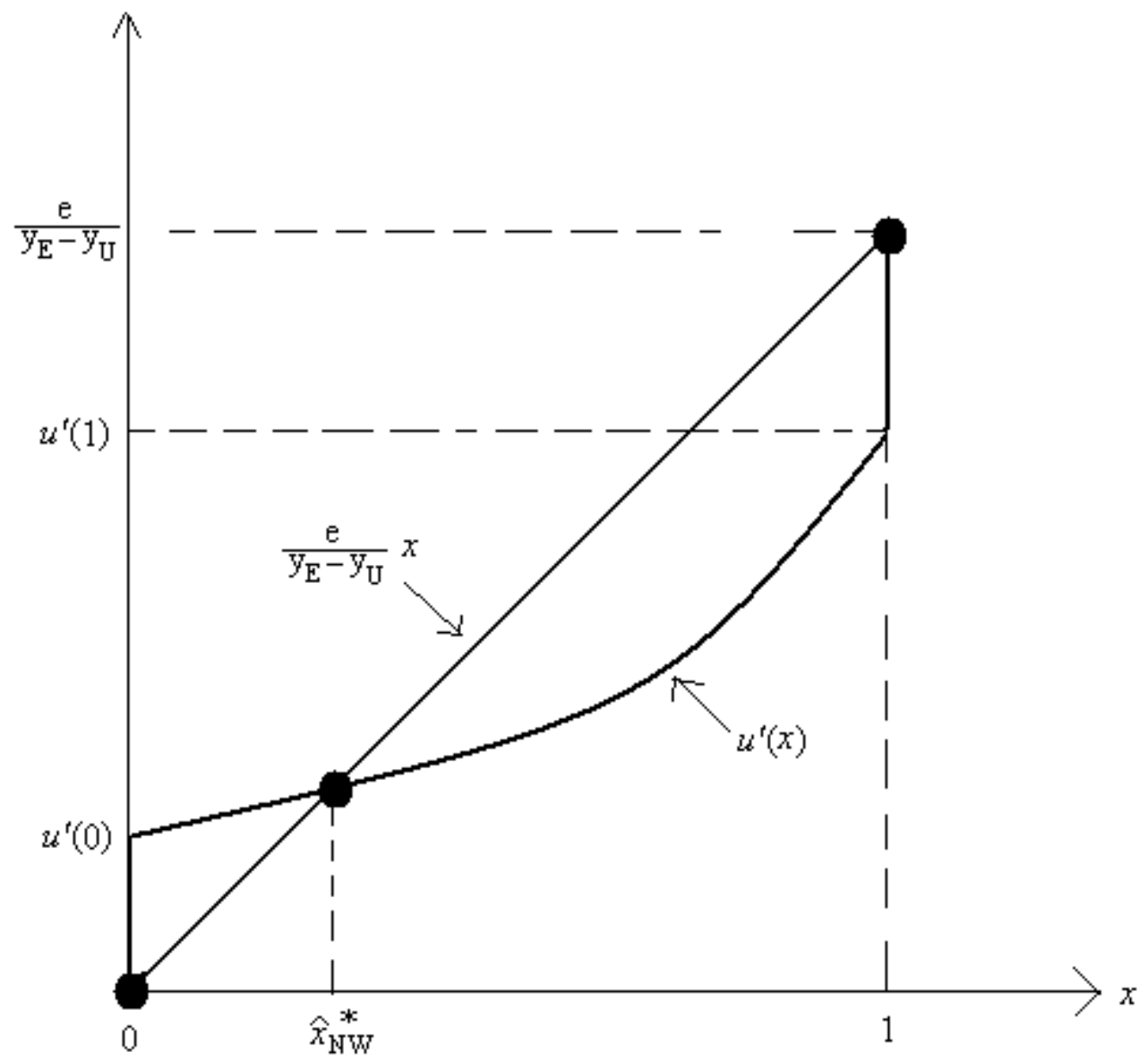

Figure 2d: Case $(i i i)$ when $e /\left(y_{E}-y_{U}\right)>u^{\prime}(1)$ 
Table 1: In many ways I think of myself as British (\% )

\begin{tabular}{lrrrrrr}
\hline & Caribbean & Indian & $\begin{array}{r}\text { African } \\
\text { Asian }\end{array}$ & Pakistani & Bangladeshi & Chinese \\
\hline Strongly agree & 20.09 & 13.98 & 25.79 & 22.53 & 14.23 & 10.00 \\
Agree & 37.77 & 43.69 & 45.28 & 37.55 & 41.90 & 38.00 \\
Neither & 8.30 & 13.40 & 10.69 & 16.21 & 20.55 & 11.00 \\
Disagree & 24.02 & 23.30 & 16.04 & 15.22 & 18.58 & 33.00 \\
Strongly & 9.83 & 5.63 & 2.20 & 8.50 & 4.74 & 8.00 \\
disagree & & & & & & \\
N & 458 & 515 & 318 & 506 & 253 & 100 \\
\hline
\end{tabular}

Table 2: If a close relative were to marry a white person (\% )

\begin{tabular}{lrrrrrr}
\hline & Caribbean & Indian & $\begin{array}{r}\text { African } \\
\text { Asian }\end{array}$ & Pakistani & Bangladeshi & Chinese \\
\hline Would not mind & 82.43 & 51.87 & 66.25 & 38.61 & 49.60 & 84.69 \\
Mind a little & 6.51 & 10.02 & 11.04 & 11.09 & 9.20 & 6.12 \\
Mind very much & 8.24 & 27.89 & 13.56 & 36.83 & 33.20 & 7.14 \\
Can't say & 2.82 & 10.22 & 9.15 & 13.47 & 8.00 & 2.05 \\
N & 461 & 509 & 317 & 505 & 250 & 98 \\
\hline
\end{tabular}

Table 3: How important is ethnicity in choosing a school? (\% )

\begin{tabular}{lrrrrrr}
\hline & Caribbean & Indian & $\begin{array}{r}\text { African } \\
\text { Asian }\end{array}$ & Pakistani & Bangladeshi & Chinese \\
\hline Very important & 15.94 & 6.81 & 8.44 & 12.06 & 16.21 & 3.03 \\
Fairly important & 20.74 & 16.15 & 15.31 & 16.60 & 18.58 & 7.07 \\
Not very important & 16.16 & 13.42 & 10.31 & 15.02 & 16.21 & 15.15 \\
No influence & 44.32 & 57.78 & 59.06 & 47.23 & 38.34 & 73.74 \\
Can't say & 2.84 & 5.84 & 6.88 & 9.09 & 10.67 & 1.01 \\
N & 458 & 514 & 320 & 506 & 253 & 99 \\
\hline
\end{tabular}

Table 4: What proportion of one's ethnic group would you like in your children's school? (\%)

\begin{tabular}{lrrrrrr}
\hline & Caribbean & Indian & $\begin{array}{r}\text { African } \\
\text { Asian }\end{array}$ & Pakistani & Bangladeshi & Chinese \\
\hline Fewer than half & 16.67 & 11.07 & 14.42 & 9.49 & 14.17 & 23.23 \\
About half & 35.06 & 18.83 & 20.06 & 28.06 & 30.31 & 7.07 \\
More than half & 4.11 & 1.55 & 1.57 & 5.14 & 5.12 & 1.01 \\
No preference & 40.69 & 63.11 & 56.11 & 48.62 & 40.94 & 68.69 \\
Can't say & 3.46 & 5.44 & 7.84 & 8.70 & 9.45 & 0.00 \\
N & 462 & 515 & 319 & 506 & 254 & 99 \\
\hline
\end{tabular}

